\title{
Cycle integrals of Maass forms of weight 0 and Fourier coefficients of Maass forms of weight 1/2
}

by

\author{
A. Biró (Budapest)
}

1. Introduction. In [K-S] Katok and Sarnak proved that the sum of the integrals of a Maass cusp form $\varphi$ of weight 0 for the group $\Gamma_{0}(1)$ on certain geodesic cycles of a given discriminant $s>0$ (or the sum of $\varphi$ over Heegner points of discriminant $s$ for $s<0$ ) equals the sum of $b_{j}(s) \overline{b_{j}(1)}$ over an orthonormal basis of the space of those Maass cusp forms of weight $1 / 2$ for $\Gamma_{0}(4)$ whose Shimura lifting (introduced in the holomorphic case in [Shim]) is $\varphi$, where $b_{j}(s)$ is the $s$ th and $b_{j}(1)$ is the first Fourier coefficient of the $j$ th weight $1 / 2$ form. Here we generalize the $s>0$ part of this result to $\Gamma_{0}(N)$ for arbitrary level $N$, and for more general products of coefficients $b_{j}(s) \overline{b_{j}(\widehat{s})}$. As a consequence (similarly to $[\mathrm{K}-\mathrm{S}]$ for $\Gamma_{0}(1)$ and the trivial character) we prove that the $L$-function of a Maass-Hecke newform for $\Gamma_{0}(N)$ twisted by a quadratic character is nonnegative at the centre of the critical strip (this was first proved in $[\mathrm{G}]$; it would be a trivial consequence of the Riemann Hypothesis for this $L$-function). In the course of proof we show that if $N$ is even, then the Shimura liftings of any Maass cusp form of weight $1 / 2$ for $\Gamma_{0}(4 N)$ are automorphic of level $N$, i.e. for $\Gamma_{0}(N)$ (and not just for $\Gamma_{0}(2 N)$ ).

The first paper studying this phenomenon was that of Maass $([\mathrm{M}])$. The holomorphic case was considered in [Shim], [Shin], [N], [Wal], [Ko1], [Ko2] and $[\mathrm{Ko}-\mathrm{Z}]$. So the holomorphic case was developed in greater generality than the theorem of Katok and Sarnak. Our result is similar to the main result of [Ko2], where the holomorphic case is considered for $\Gamma_{0}(N)$, but $N$ is restricted to be odd and squarefree.

Our method of proof is completely different from the proofs in the abovementioned papers; the idea of our proof is briefly discussed in Section 3. We do not use theta-functions and kernel functions for the Shimura lifting. Our proof is based on the idea of [B] (but we do not refer to that paper, the relevant formula is developed in Section 5) and on Kuznetsov's formula

2000 Mathematics Subject Classification: Primary 11F72. 
for half-integral weight Maass forms, which is worked out in $[\mathrm{P}]$, and for Kohnen's subspace in the Appendix of the present paper. We will also need a discrete Fourier transform duality relation proved in [Ko2], Proposition 5. During our proof we obtain automatically the fact that the Shimura liftings produce automorphic forms. The only reason why we consider only the case $s>0$ is that Kuznetsov's formula in $[\mathrm{P}]$ is worked out only for sums $\sum b(m) \overline{b(n)}$ with $m, n>0$, and (as far as I know) the case $m n<0$ has not been worked out yet.

Because of the presence of Kuznetsov's formula we need several lemmas for special functions; we prove them in Section 9.

2. Notations and statement of results. We denote by $H$ the upper half-plane, $\operatorname{SL}(2, \mathbb{R})$ is the group of $2 \times 2$ real matrices with determinant 1 , $\mathrm{GL}^{+}(2, \mathbb{R})$ denotes the group of $2 \times 2$ real matrices with positive determinant. If $M$ is a positive integer, then $\Gamma_{0}(M)$ is the group of $2 \times 2$ integer matrices with determinant 1 and lower left entry divisible by $M$, and

$$
\widehat{\Gamma}_{0}(M)=\Gamma_{0}(M) /\left\{\left(\begin{array}{ll}
1 & 0 \\
0 & 1
\end{array}\right),\left(\begin{array}{cc}
-1 & 0 \\
0 & -1
\end{array}\right)\right\} .
$$

If $a$ is a residue class $\bmod q,(a, q)=1$, then $\bar{a}$ will denote the residue class $\bmod q$ with $a \bar{a} \equiv 1(\bmod q)$. The meaning of $\bar{a}$ (i.e. the modulus $q$ ) will always be clear from the context. We write $e(x)$ for $e^{2 \pi i x}$.

If $n \neq 0$ is an integer, then $n \mid x$ means that $x$ is an integer and $n$ divides $x$. If $n$ is a prime power, $n \| x$ means that $x$ is an integer and $n$ is an exact divisor of $x$.

A fundamental discriminant is an integer $D$ which is a product of relatively prime factors of the form

$$
-4,8,-8 \text { or }(-1)^{(p-1) / 2} p \quad(p>2) .
$$

(The product may be empty, so we understand $D=1$ to be a fundamental discriminant.) Then $(\underline{D})$ is a primitive character $\bmod |D|$ (see p. 40 of [Da]).

For $g=\left(\begin{array}{ll}a & b \\ c & d\end{array}\right) \in \mathrm{GL}^{+}(2, \mathbb{R})$ let $j_{g}(z)=(c z+d) / \sqrt{\operatorname{det} g}$. Define the slash operator (of weight $1 / 2$ ) acting on functions $f: H \rightarrow \mathbb{C}$ by

$$
(f \mid g)(z)=\frac{j_{g}(z)^{-1 / 2}}{\left|j_{g}(z)^{-1 / 2}\right|} f(g z) .
$$

For $\gamma=\left(\begin{array}{ll}a & b \\ c & d\end{array}\right) \in \Gamma_{0}(4)$ let $\nu(\gamma)=\bar{\varepsilon}_{d}\left(\frac{c}{d}\right)$, where $\left(\frac{c}{d}\right)$ has its usual meaning (we summarize its definition and basic properties in the Appendix, Section A.1), and

$$
\varepsilon_{d}= \begin{cases}1 & \text { if } d \equiv 1(\bmod 4) \\ i & \text { if } d \equiv-1(\bmod 4)\end{cases}
$$


If $N$ is a positive integer, we say that a function $f$ on $H$ is a Maass form of weight $1 / 2$ for the group $\Gamma_{0}(4 N)$ if $f \mid \gamma=\nu(\gamma) f$ for every $\gamma \in \Gamma_{0}(4 N)$, $f$ is an eigenfunction of the operator

$$
\Delta_{1 / 2}=y^{2}\left(\frac{\partial^{2}}{\partial x^{2}}+\frac{\partial^{2}}{\partial y^{2}}\right)-\frac{1}{2} i y \frac{\partial}{\partial x}
$$

of eigenvalue $\lambda \leq 0$ (so $\Delta_{1 / 2} f=\lambda f$ ), and $f$ has at most polynomial growth at cusps. It is known that then it has a Fourier development at $\infty$ of the form

$$
f(z)=c_{f, \infty}(y)+\sum_{n \neq 0} b_{f, \infty}(n) W_{\frac{1}{4} \operatorname{sgn}(n), i t}(4 \pi|n| y) e(n x),
$$

where $W$ is the Whittaker function (see [M-O], Chapter 7), $1 / 4+t^{2}=-\lambda$, and $c_{f, \infty}(y)$ is a linear combination of $y^{1 / 2+i t}$ and $y^{1 / 2-i t}$.

Let us introduce the notation $V=V_{1 / 2}(4 N)$ for the Hilbert subspace generated by Maass forms of the Hilbert space of complex-valued functions $f$ on $H$ with $f \mid \gamma=\nu(\gamma) f$ for every $\gamma \in \Gamma_{0}(4 N)$, and $\int_{\Gamma_{0}(4 N) \backslash H}|f|^{2} d x d y / y^{2}$ $<\infty$. It is well known that if $f \in V_{1 / 2}(4 N)$ is a Maass form, then it has eigenvalue $\lambda \leq-3 / 16$, and if $\lambda<-3 / 16$, then $f$ is a cusp form, i.e. the zeroth Fourier coefficients of $f$ at all cusps vanish.

Definition. For a cusp form $f \in V_{1 / 2}(4 N)$ with $\Delta_{1 / 2}$-eigenvalue $\lambda=$ $-1 / 4-t^{2}$ and a fundamental discriminant $D>0$ we define the Dth Shimura lifting, $\mathrm{Sh}_{D} f$, of $f$ by

$$
\operatorname{Sh}_{D} f(z)=\sum_{k \neq 0} a_{\operatorname{Sh}_{D} f}(k) W_{1 / 2+2 i t}(k z),
$$

where $W_{s}(z)$ is defined as in [I1], p. 22, and

$$
a_{\mathrm{Sh}_{D} f}(k)=\sum_{\substack{P Q=k \\ P>0,(N, P)=1}} \frac{|Q|^{1 / 2}}{P}\left(\frac{D}{P}\right) b_{f, \infty}\left(D Q^{2}\right) .
$$

If $N$ is odd, let $V^{+}=V_{1 / 2}^{+}(4 N)$ be the subspace of $V$ generated by Maass forms $f$ with $b_{f, \infty}(n)=0$ for $n \equiv 2,3(\bmod 4)$ (Kohnen's subspace; see [Ko1], [Ko2] in the holomorphic case). The space $V^{+}$is the eigenspace in $V$ with eigenvalue 1 of the operator

$$
L f=L_{4 N} f=\frac{1}{2(1+i)} \sum_{w=0}^{3}\left(f \mid\left(\begin{array}{cc}
1 & 1 / 4 \\
0 & 1
\end{array}\right)\right) \mid\left(\begin{array}{cc}
1 & 0 \\
4 N w & 1
\end{array}\right)
$$

(see Kohnen's papers and also our Appendix). Define the space $V_{1 / 2}^{*}(4 N)$ by

$$
V_{1 / 2}^{*}(4 N)= \begin{cases}V_{1 / 2}(4 N) & \text { for } N \text { even } \\ V_{1 / 2}^{+}(4 N) & \text { for } N \text { odd }\end{cases}
$$


THEOREM 1. If $f \in V_{1 / 2}^{*}(4 N)$ is a cusp form with $\Delta_{1 / 2}$-eigenvalue $\lambda<-3 / 16, \lambda=-1 / 4-t^{2}$, then $\mathrm{Sh}_{D} f$ is a cusp form for $\Gamma_{0}(N)$ with $\Delta$-eigenvalue $-1 / 4-(2 t)^{2}$.

For $N$ even this is the result stated in the introduction.

We remark that in the holomorphic case Shimura proved that his liftings produce automorphic forms of integral weight (see [Shim]), and it was proved later in [N] that forms of level $4 N$ are always mapped to level $2 N$. It was proved in [Ko1], [Ko2] that the Shimura liftings map Kohnen's subspace to cusp forms of level $N$ for $N$ odd, and not just to level $2 N$ (this is the significance of Kohnen's subspace).

Theorem 1 shows that for $N$ even all forms of level $4 N$ are always mapped to level $N$. If $N$ is odd, the theorem is the exact analogue of Kohnen's result.

If $Q(X, Y)=a X^{2}+b X Y+c Y^{2}$ is a quadratic form with integer coefficients, $d=b^{2}-4 a c$ is its discriminant, $d \neq 0$, and $D$ is a fundamental discriminant with $D \mid d$ and $d / D \equiv 0,1(\bmod 4)$, define

$$
\omega_{D}(Q)= \begin{cases}0 & \text { if }(a, b, c, D)>1, \\ \left(\frac{D}{r}\right) & \text { if }(a, b, c, D)=1,\end{cases}
$$

where $r$ is any number represented by $Q$ with $(r, D)=1$. The symbol $\omega_{D}(Q)$ is well defined, and it depends only on the $\operatorname{SL}(2, \mathbb{Z})$-equivalence class of $Q$ (see $[$ Ko2]).

For an integer matrix $\gamma=\left(\begin{array}{ll}a & b \\ c & d\end{array}\right), t=a+d, n=a d-b c, s=t^{2}-4 n$, $s \neq 0$, and a fundamental discriminant $D$ with $D \mid s$ and $s / D \equiv 0,1(\bmod 4)$, define

$$
\omega_{D}(\gamma)=\omega_{D}\left((-Y X)\left(\begin{array}{ll}
a & b \\
c & d
\end{array}\right)\left(\begin{array}{l}
X \\
Y
\end{array}\right)\right)=\omega_{D}\left(c X^{2}+(d-a) X Y-b Y^{2}\right) .
$$

If $\delta \in \mathrm{SL}(2, \mathbb{Z})$, then $\omega_{D}\left(\delta^{-1} \gamma \delta\right)=\omega_{D}(\gamma)$.

If $N$ is a positive integer, and $s$ is a positive integer with $s \equiv 0,1$ $(\bmod 4)$, let

$Q_{N, s}=\left\{Q(X, Y)=A X^{2}+B X Y+C Y^{2}: A, B, C \in \mathbb{Z}, N \mid A, s=B^{2}-4 A C\right\}$.

If $Q(X, Y)$ and $\widetilde{Q}(X, Y)$ are quadratic forms, we say that they are equivalent over $\Gamma_{0}(N)$ if $\widetilde{Q}(X, Y)=Q(\tilde{X}, \tilde{Y})$, where $\left(\begin{array}{c}\widetilde{X} \\ \widetilde{Y}\end{array}\right)=\tau\left(\begin{array}{c}X \\ Y\end{array}\right)$ with some $\tau \in \Gamma_{0}(N)$. Denote by $\Lambda_{N, s}$ a complete set of representatives of the $\Gamma_{0}(N)$ equivalence classes of $Q_{N, s}$. It is well known that $\Lambda_{N, s}$ is a finite set.

Definition. For $Q \in Q_{N, s}, Q(X, Y)=A X^{2}+B X Y+C Y^{2}$, if $z_{1}$ and $z_{2}$ are the roots of $A z^{2}+B z+C$ (if $A=0$, one root is $\infty$ ), let $C_{Q, N}$ be the noneuclidean line connecting $z_{1}$ and $z_{2}$ factorized by the action of $\left\{\gamma \in \Gamma_{0}(N): \gamma z_{1}=z_{1}, \gamma z_{2}=z_{2}\right\}$. 
For $p$ a prime with $(p, 4 N)=1$, introduce the weight 0 Hecke operators $H_{p}$ by

$$
\left(H_{p} F\right)(z)=\frac{1}{\sqrt{p}}\left(\sum_{k=0}^{p-1} F\left(\frac{z+k}{p}\right)+F(p z)\right) .
$$

For the weight $1 / 2$ Hecke operators $T_{p^{2}}$ see [Shim].

Let $\left\{f_{j}(z)\right\}_{j=0}^{\infty}$ be a Maass form orthonormal basis of $V_{1 / 2}(4 N)$ such that every $f_{j}$ is an eigenfunction of $T_{p^{2}}$ for every $(p, 4 N)=1$, and for $N$ odd every $f_{j}$ is an eigenfunction of $L$. This is possible (see [Ko3] and our Appendix), since the Hecke operators $T_{p^{2}}$ for $(p, 4 N)=1$, and the operator $L$ for $N$ odd, are self-adjoint, they commute with each other and with $\Delta_{1 / 2}$.

Define

$$
p_{N}= \begin{cases}1 & \text { for } N \text { even, } \\ 3 / 2 & \text { for } N \text { odd } .\end{cases}
$$

Our main result is the following.

Theorem 2. Let $\varphi$ be a normalized Maass-Hecke newform for $\Gamma_{0}(N)$ with $L_{2}$-norm $\langle\varphi, \varphi\rangle$, let $s>0$ be an integer with $s \equiv 0,1(\bmod 4)$, and $D>0$ a fundamental discriminant with $D \mid s$ and $s / D \equiv 0,1(\bmod 4)$. Then $(d S=|d z| / y$ is the hyperbolic arc length $)$

$$
\begin{aligned}
\frac{1}{\langle\varphi, \varphi\rangle}\left(\sum_{Q \in \Lambda_{N, s}} \omega_{D}(Q)\right. & \left.\int_{C_{Q, N}} \varphi d S\right) \\
& =8 p_{N} \sqrt{\pi} s^{3 / 4} \sum_{\substack{f_{j} \in V_{1 / 2}^{*}(4 N) \\
\operatorname{Sh}_{D} f_{j}=b_{j, \infty}(D) \varphi}} b_{j, \infty}\left(\frac{s}{D}\right) \bar{b}_{j, \infty}(D) .
\end{aligned}
$$

Note that if $\varphi$ is odd, then both sides are 0 .

COROllary. Let $\varphi$ be an even normalized Maass-Hecke newform for $\Gamma_{0}(N)$ with $\Delta$-eigenvalue $\lambda=-1 / 4-t^{2}$ and Fourier expansion $\varphi(z)=$ $\sum_{k \neq 0} a(k) W_{1 / 2+i t}(k z)$, so $a(1)=1$ and $a(k)=a(-k)$. For a fundamental discriminant $D>0$ with $(D, N)=1$ define the L-function $L(\varphi, D ; s)$ by holomorphic continuation of the series

$$
\sum_{k=1}^{\infty}(a(k) \sqrt{k})\left(\frac{D}{k}\right) k^{-s}
$$

Then $L(\varphi, D ; 1 / 2) \geq 0$.

As mentioned in the introduction, we need a Kuznetsov-type formula for Kohnen's subspace (more precisely, for the space $V_{1 / 2}^{*}(4 N)$ ). This formula (Theorem B) is stated in the next section, and proved in the Appendix. 
3. The idea of the proof and preliminaries. Let $m$ be a smooth, compactly supported real-valued function on $(0, \infty)$ (i.e. it vanishes in a neighbourhood of $\infty$ and in a neighbourhood of 0$)$, and

$$
m(z, w)=m\left(\frac{|z-w|^{2}}{\operatorname{Im} z \operatorname{Im} w}\right) .
$$

Then $m$ is a point-pair invariant, so $m(g z, g w)=m(z, w)$ for every $g \in$ $\mathrm{GL}^{+}(2, \mathbb{R})$. Let $t, n$ be given positive integers with $s=t^{2}-4 n>0$. Observe that if we choose $t$ and $n$ suitably, then $s$ may be any positive integer with $s \equiv 0,1(\bmod 4)$. Let $D>0$ be a fundamental discriminant with $D \mid s$ and $s / D \equiv 0,1(\bmod 4)$. For $z \in H$ define

$$
M(N, z)=\sum_{\gamma \in \Gamma_{N}} \omega_{D}(\gamma) m(z, \gamma z),
$$

where $N$ is a positive integer, and

$$
\Gamma_{N}=\Gamma_{n, t, N}=\left\{\left(\begin{array}{ll}
a & b \\
c & d
\end{array}\right): a, b, c, d \in \mathbb{Z}, N \mid c, a d-b c=n, a+d=t\right\} .
$$

Of course $M(N, z)$ also depends on $t, n$ and $D$, and $\Gamma_{N}$ depends on $t$ and $n$; but $t, n$ and $D$ are fixed, while $N$ will vary (among the divisors of a fixed integer), so we only indicate the dependence on $N$.

Then for $\delta \in \Gamma_{0}(N)$, using $m(\delta z, \gamma \delta z)=m\left(z, \delta^{-1} \gamma \delta z\right), \delta^{-1} \Gamma_{N} \delta=\Gamma_{N}$, and $\omega_{D}(\gamma)=\omega_{D}\left(\delta^{-1} \gamma \delta\right)$, we have

$$
M(N, \delta z)=\sum_{\gamma \in \Gamma_{N}} \omega_{D}\left(\delta^{-1} \gamma \delta\right) m\left(z, \delta^{-1} \gamma \delta z\right)=M(N, z) .
$$

Our identity in Theorem 2 will follow by computing $M(N, z)$ in two different ways. We now describe these two ways briefly.

For the first computation of $M(N, z)$ observe that

$$
z-\gamma z=\frac{c z^{2}+(d-a) z-b}{c z+d} \quad \text { for } \gamma=\left(\begin{array}{ll}
a & b \\
c & d
\end{array}\right)
$$

so

$$
M(N, z)=\sum_{\gamma=\left(\begin{array}{c}
a b \\
c d
\end{array}\right) \in \Gamma_{N}} \omega_{D}(\gamma) m\left(\frac{\left|c z^{2}+(d-a) z-b\right|^{2}}{n \operatorname{Im}^{2} z}\right) .
$$

The condition $a d-b c=n$ is equivalent to $s-r^{2}=(4 c) b$ (with the notations $s=t^{2}-4 n, r=a-d$ ), so (since the sum with $c<0$ is the same as that with $c>0$ in view of the pairing $\left(\begin{array}{ll}a & b \\ c & d\end{array}\right) \leftrightarrow\left(\begin{array}{cc}d & -b \\ -c & a\end{array}\right)$ and $\left.D>0\right)$ we have

$$
M(N, z)=M_{0}(N, z)+2 M_{+}(N, z),
$$

where 


$$
\begin{aligned}
& M_{+}(N, z) \\
& =\sum_{\substack{r, c \\
N|c, c>0,4 c| s-r^{2}}} \omega_{D}\left(c X^{2}-r X Y+\frac{r^{2}-s}{4 c} Y^{2}\right) m\left(\left|c z^{2}-r z+\frac{r^{2}-s}{4 c}\right|^{2} / n \operatorname{Im}^{2} z\right),
\end{aligned}
$$

and

$$
M_{0}(N, z)=\sum_{\gamma \in \Gamma_{N}, \gamma \infty=\infty} \omega_{D}(\gamma) m(z, \gamma z),
$$

which corresponds to $c=0$. (Note that $M_{0}(N, z)=0$ if $s$ is not a square, because if $c=0$, then $s=(a-d)^{2}$.) We will apply the Poisson formula in $r$ for $M_{+}(N, z)$. In this way (Kohnen-) Kloosterman sums for weight $1 / 2$ come into play by using the discrete Fourier transform identity (Theorem A below), and we will have a possibility of applying Kuznetsov's formula for (Kohnen's subspace of) weight 1/2 Maass forms (see Theorem B below). So this first computation of $M(N, z)$ leads us to Fourier coefficients of Maass forms of weight $1 / 2$.

For the second computation of $M(N, z)$ we will consider it as an automorphic function for $\Gamma_{0}(N)$ and determine its spectral expansion. One needs for this the inner product of $M(N, z)$ against cusp forms of weight 0 (see Section 5), and (as in [B]) this leads to cycle integrals of Maass forms of weight 0 .

Already now we mention that in proving the basic Lemma 10 we will eventually compute in these two ways the function $I_{m, \tau}(A)$ defined before Lemma 9 (the functions $M^{*}(z)$ and $G(y)$ are also defined there), where $\tau \geq 0, T_{0} \neq 0$ is an integer, and $\operatorname{Re} A>-1 / 2$ (moreover, at first $A$ will be large and positive). We will fix $t, n, D, \tau, N$ and $T_{0}$ (but after Lemma 10, $T_{0}, t$ and $n$ (hence $s$ ) will vary) and let $m$ tend to "Dirac's delta" of the point $s / n$, and $A \rightarrow-1 / 2+0$.

We now state two theorems needed for the first computation of $M(N, z)$. We define the Kohnen-Kloosterman sums $S_{K}(m, n ; c)$ in the following way. If $m$ and $n$ are integers, and $4 \mid c$, let

$$
S_{\infty, \infty}(m, n ; c)=\sum_{\substack{x(\bmod c) \\(x, c)=1}} \varepsilon_{x}\left(\frac{c}{x}\right) e\left(\frac{m \bar{x}+n x}{c}\right) .
$$

For $m \equiv 0,1(\bmod 4), n \equiv 0,1(\bmod 4)$ define

$$
S_{K}(m, n ; c)= \begin{cases}S_{\infty, \infty}(m, n ; c) & \text { if } 8 \mid c, \\ 2 S_{\infty, \infty}(m, n ; c) & \text { if } 4 \| c,\end{cases}
$$

and $S_{K}(m, n ; c)=0$ if 4 does not divide $c$. Let us mention that for the proof of Theorems 1 and 2 we do not need this concrete shape of $S_{K}(m, n ; c)$, but just the information contained in Theorems A and B below. 
The following theorem is proved in [Ko2], Proposition 5.

Theorem A. Let $c>0$ be an integer, $s \equiv 0,1(\bmod 4), s \neq 0$, and let $D>0$ be a fundamental discriminant with $D \mid s$ and $s / D \equiv 0,1(\bmod 4)$. For any integer $T$ define

$$
F(T)=F_{c, s, D}(T)=\sum_{\substack{r(\bmod 4 c) \\ 4 c \mid s-r^{2}}} e\left(\frac{T r}{4 c}\right) \omega_{D}\left(c X^{2}-r X Y+\frac{r^{2}-s}{4 c} Y^{2}\right) .
$$

Then

$$
F(T)=(1-i) \sum_{\substack{\widehat{c}|4 c \\ 2(4 c / \widehat{c})| T}}\left(\frac{D}{4 c / \widehat{c}}\right) \frac{1}{\widehat{c}^{1 / 2}} S_{K}\left(\frac{s}{D}, D\left(\frac{\widehat{T}}{2}\right)^{2} ; \widehat{c}\right),
$$

where $\widehat{T}=T /(4 c / \widehat{c})$.

Let $\left\{f_{j}(z)\right\}_{j=0}^{\infty}$ be a Maass form orthonormal basis of $V_{1 / 2}(4 N)$, where for $N$ odd every $f_{j}$ is an eigenfunction of $L, \Delta_{1 / 2} f_{j}=\lambda_{j} f_{j}, 1 / 4+t_{j}^{2}=-\lambda_{j}$ with $t_{j} \geq 0$ or $i t_{j} \geq 0$, and denote the Fourier coefficients at a cusp $a$ by $b_{j, a}(n)=b_{f_{j}, a}(n)$. Let $\left\{\kappa_{1}, \ldots, \kappa_{h}\right\}$ be a complete set of inequivalent singular cusps (see Section A.1) for the multiplier system $\nu$ and group $\Gamma_{0}(4 N)$. For each $j=1, \ldots, h$ define the Eisenstein series $E_{j}(z, s, 4 N)$ for the group $\Gamma_{0}(4 N)$, weight $1 / 2$, multiplier system $\nu$, and cusp $\kappa_{j}$ in the usual way (see $[\mathrm{P}],[\mathrm{Du}]$ ), and for $n \neq 0$ and a cusp $a$ define $\varphi_{j, n, a}(s)$ such that

$$
\frac{\pi^{s} e(-1 / 8)\left|n-\chi_{a}\right|^{s-1}}{\Gamma\left(s+\frac{1}{4} \operatorname{sgn}\left(n-\chi_{a}\right)\right)} \varphi_{j, n, a}(s)
$$

is the $n$th Fourier coefficient of $E_{j}(z, s, 4 N)$ at $a$ (see Section A.1 for the definition of $\chi_{a}$ and the Fourier expansion of a Maass form of weight $1 / 2$ at a cusp).

Now let $\varphi(x)$ be a smooth function on $[0, \infty)$ such that $\varphi(0)=\varphi^{(1)}(0)=0$, and for some $\varepsilon>0, \varphi(x)=O\left(x^{-1-\varepsilon}\right)$ and $\varphi^{(l)}(x)=O\left(x^{-2-\varepsilon}\right)$ for $l=1,2,3$ as $x \rightarrow \infty$. Define the Kuznetsov transform of $\varphi$ by

$$
\widetilde{\varphi}(t)=\int_{0}^{\infty} J_{t-1}(x) \varphi(x) \frac{d x}{x}
$$

and

$$
\widehat{\varphi}(t)=\beta(t)\left(\varphi^{*}(t)-\varphi^{*}(-t)\right)
$$

where

$$
\begin{aligned}
\beta(t) & =\frac{\pi^{2} e(3 / 8)}{(\operatorname{sh} \pi t)(\operatorname{ch} 2 \pi t) \Gamma(1 / 4+i t) \Gamma(1 / 4-i t)}, \\
\varphi^{*}(t) & =\cos \pi(1 / 4+i t) \int_{0}^{\infty} J_{2 i t}(x) \varphi(x) \frac{d x}{x} .
\end{aligned}
$$


If $N$ is odd, let $q$ and $s$ be the cusps of $\Gamma_{0}(4 N)$ given by

$$
\begin{aligned}
& q= \begin{cases}(N+1) /(4 N) & \text { if } N \equiv 1(\bmod 4), \\
(3 N+1) /(12 N) & \text { if } N \equiv 3(\bmod 4) ;\end{cases} \\
& s= \begin{cases}(3 N+1) /(12 N) & \text { if } N \equiv 1(\bmod 4), \\
(N+1) /(4 N) & \text { if } N \equiv 3(\bmod 4) .\end{cases}
\end{aligned}
$$

We will prove the following theorem in the Appendix.

Theorem B. Let $m, n \geq 1$ be positive integers, $m, n \equiv 0,1(\bmod 4)$, let $N$ be a positive integer,

$$
\sum=\sum_{\substack{c>0 \\ N \mid c}} \frac{S_{K}(m, n ; c)}{c} \varphi\left(4 \pi \frac{\sqrt{m n}}{c}\right)
$$

let $\varphi$ and $\left\{f_{j}(z)\right\}_{j=0}^{\infty}$ be as above, and assume that $\widetilde{\varphi}(1 / 2+2 j)=0$ for $j=1,2 \ldots$ Then

$$
\sum=4 p_{N} \sqrt{m n} \sum_{f_{j} \in V_{1 / 2}^{*}(4 N)} \frac{\bar{b}_{j, \infty}(m) b_{j, \infty}(n)}{\operatorname{ch}\left(\pi t_{j}\right)} \widehat{\varphi}\left(t_{j}\right)+E,
$$

where $E$ is a "remainder term", the Eisenstein series part, more precisely $E=\sum_{j=1}^{h} \int_{-\infty}^{\infty}\left(\frac{n}{m}\right)^{i t} \bar{\varphi}_{j, m, \infty}(1 / 2+i t) \varphi_{j, n, \infty / K}(1 / 2+i t) \frac{\widehat{\varphi}(t)}{\operatorname{ch}(\pi t)|\Gamma(3 / 4+i t)|^{2}} d t$,

where

$$
\begin{aligned}
& \varphi_{j, n, K}(1 / 2+i t) \\
& = \begin{cases}\varphi_{j, n, \infty}(1 / 2+i t)+\frac{1}{1+i} 4^{-i t} \varphi_{j, n / 4, s}(1 / 2+i t) & \text { if } n \equiv 0(\bmod 4), \\
\varphi_{j, n, \infty}(1 / 2+i t)+\frac{1}{1+i} 4^{-i t} \varphi_{j,(n+3) / 4, q}(1 / 2+i t) & \text { if } n \equiv 1(\bmod 4),\end{cases}
\end{aligned}
$$

and $\varphi_{j, n, \infty / K}(1 / 2+i t)$ is $\varphi_{j, n, \infty}(1 / 2+i t)$ for $N$ even, and $\varphi_{j, n, K}(1 / 2+i t)$ for $N$ odd.

4. The first way of computing $M(N, z)$. We now continue the first computation of $M(N, z)$, started in the previous section. We will need the following lemma.

Lemma 1. If $c>0, r$ and $s$ are real, and $z$ is a complex number, then

$$
\left|c z^{2}-r z+\frac{r^{2}-s}{4 c}\right|^{2}=s \operatorname{Im}^{2} z+\left(\frac{s}{4 c}-c\left(\left(\operatorname{Re} z-\frac{r}{2 c}\right)^{2}+\operatorname{Im}^{2} z\right)\right)^{2} .
$$

Proof. It is easy to see that we may assume $r=0$, and then $c=1$, and the statement with $r=0$ and $c=1$ follows from $\frac{1}{4}\left(z^{2}+\bar{z}^{2}\right)=\frac{1}{2}|z|^{2}-\operatorname{Im}^{2} z$. 
This shows, in particular, that for $c$ large enough the $m$-function in $M_{+}(N, z)$ is 0 .

We apply the Poisson formula in $r$ for $M_{+}(N, z)$. In general, a trivial consequence of the Poisson formula is that if $c(n)$ is periodic with respect to $p$, where $p$ is a positive integer, then

$$
\sum_{n=-\infty}^{\infty} c(n) f(n)=\sum_{n=-\infty}^{\infty} \widehat{c}_{p}(n) \widehat{f}\left(\frac{2 \pi n}{p}\right),
$$

where $\widehat{f}$ is the Fourier transform of $f$ and $\widehat{c}_{p}$ is the discrete Fourier transform of $c$, i.e.

$$
\widehat{f}(t)=\int_{-\infty}^{\infty} f(y) e^{-i t y} d y \quad \text { and } \quad \widehat{c}_{p}(n)=\frac{1}{p} \sum_{a(\bmod p)} e\left(\frac{n a}{p}\right) c(a) .
$$

By Theorem A this gives (with $p=4 c$ )

where

$$
\begin{aligned}
M_{+}(N, z)= & \sum_{c>0, N \mid c} \sum_{2 \mid T}\left(\frac{1-i}{4 c} \sum_{\substack{\widehat{c}|4 c \\
2(4 c / \widehat{c})| T}}\left(\frac{D}{4 c / \widehat{c}}\right) \frac{1}{\widehat{c}^{1 / 2}}\right. \\
& \left.\times S_{K}\left(\frac{s}{D}, D\left(\frac{\widehat{T}}{2}\right)^{2} ; \widehat{c}\right)\right) m^{*}(c, T, z),
\end{aligned}
$$

$m^{*}(c, T, z)=\int_{-\infty}^{\infty} m\left(\left|c z^{2}-r z+\frac{r^{2}-s}{4 c}\right|^{2} / n \operatorname{Im}^{2} z\right) e\left(\frac{-T r}{4 c}\right) d r, \quad \widehat{T}=\frac{T}{4 c / \widehat{c}}$.

We would like to apply Kuznetsov's formula, so we need the condition $4 N \mid \widehat{c}$, but at the moment we only have $N \mid c$ and $4 \mid \widehat{c}$. This explains the definition

$$
M_{+}^{*}(z)=\sum_{L \mid N} \frac{\left(\frac{D}{L}\right)}{L} \mu(L) M_{+}(N / L, L z),
$$

where $\mu$ is the Möbius function. Since $m^{*}(c, T, z)=m^{*}(c / L, T / L, L z)$, we have

$$
\begin{aligned}
M_{+}(N / L, L z)= & \sum_{c>0, N \mid c} \sum_{2 L \mid T}\left(\frac{1-i}{4 c / L} \sum_{\substack{\widehat{c} \mid 4 c / L \\
2(4 c / \widehat{c} \mid T}}\left(\frac{D}{4 c / L \widehat{c}}\right) \frac{1}{\widehat{c}^{1 / 2}}\right. \\
& \left.\times S_{K}\left(\frac{s}{D}, D\left(\frac{\widehat{T}}{2}\right)^{2} ; \widehat{c}\right)\right) m^{*}(c, T, z),
\end{aligned}
$$

where $\widehat{T}=T /(4 c / \widehat{c})(=(T / L) /(4(c / L) / \widehat{c}))$. Here we can replace the condition $2 L \mid T$ by $2 \mid T$, since $L \mid 4 c / \widehat{c}$. Because

$$
\left(\frac{D}{L}\right)\left(\frac{D}{4 c / L \widehat{c}}\right)=\left(\frac{D}{4 c / \widehat{c}}\right)
$$


and $\sum_{L|N, L| 4 c / \widehat{c}} \mu(L)$ is 1 for $(N, 4 c / \widehat{c})=1$, and 0 otherwise, we have

$$
\begin{aligned}
M_{+}^{*}(z)= & \sum_{c>0, N \mid c} \sum_{2 \mid T}\left(\frac{1-i}{4 c} \sum_{\substack{\widehat{c}|4 c,(N, 4 c / \widehat{c})=1 \\
2(4 c / \widehat{c})| T}}\left(\frac{D}{4 c / \widehat{c}}\right) \frac{1}{\widehat{c}^{1 / 2}}\right. \\
& \left.\times S_{K}\left(\frac{s}{D}, D\left(\frac{\widehat{T}}{2}\right)^{2} ; \widehat{c}\right)\right) m^{*}(c, T, z) .
\end{aligned}
$$

In $M_{+}^{*}(z)$ we fix $P=4 c / \widehat{c}, Q=\widehat{T} / 2$ getting

$$
\begin{aligned}
M_{+}^{*}(z)= & \sum_{P>0,(N, P)=1} \frac{1-i}{P}\left(\frac{D}{P}\right) \sum_{Q} \sum_{\widehat{c}>0,4 N \mid \widehat{c}} \frac{1}{\widehat{c}^{3 / 2}} \\
& \times S_{K}\left(\frac{s}{D}, D Q^{2} ; \widehat{c}\right) m^{*}(c, T, z),
\end{aligned}
$$

where $T=2 P Q, c=\widehat{c} P / 4$. Indeed, $N|c, 4| \widehat{c},(N, P)=1$ implies $N \mid \widehat{c} / 4$.

In $m^{*}(c, T, z)$ we put $R=\pi T(r /(2 c)-\operatorname{Re} z)$ and

$$
\frac{\sin \vartheta}{\cos \vartheta}=\frac{1}{\sqrt{s} \operatorname{Im} z}\left(\frac{s}{4 c}-c\left(\left(\frac{R}{\pi T}\right)^{2}+\operatorname{Im}^{2} z\right)\right) .
$$

Then by Lemma 1 , for $T \neq 0$ we get

$$
m^{*}(c, T, z)=e^{-i \pi T \operatorname{Re} z} \frac{2 c}{\pi|T|} \int_{-\infty}^{\infty} m\left(\frac{s}{n \cos ^{2} \vartheta}\right) e^{-i R} d R .
$$

We observe on the other hand that $m^{*}(c, 0, z)$ depends only on $\operatorname{Im} z$. This means that if $T_{0} \neq 0$ is a fixed integer, then setting

$$
\begin{aligned}
M_{+}^{*}\left(y, T_{0}\right) & =\int_{0}^{1} M_{+}^{*}(\xi+i y) e\left(T_{0} \xi\right) d \xi, \\
\varphi_{y}(x) r & =x^{1 / 2} m^{*}\left(\frac{\pi|T| \sqrt{s}}{2 x}, T, i y\right)
\end{aligned}
$$

for $x>0, y>0$ (in order to apply Kuznetsov's formula), we have

$$
\begin{aligned}
M_{+}^{*}\left(y, T_{0}\right)= & \frac{1-i}{2 \sqrt{\pi} s^{1 / 4}} \sum_{\substack{P Q=T_{0} \\
P>0,(N, P)=1}} \frac{\left(\frac{D}{P}\right)}{P} \cdot \frac{1}{|Q|^{1 / 2}} \\
& \times \sum_{\widehat{c}>0,4 N \mid \widehat{c}} \frac{1}{\widehat{c}} S_{K}\left(\frac{s}{D}, D Q^{2} ; \widehat{c}\right) \varphi_{y}\left(4 \pi \frac{\sqrt{s}|Q|}{\widehat{c}}\right) .
\end{aligned}
$$

Observe that then $T=2 T_{0}$, since $T=2 P Q, T_{0}=P Q$. 
In order to avoid convergence problems we take an integral of the form

$$
I_{m, G}^{+}=\int_{0}^{\infty} G(|\pi T| y) M_{+}^{*}\left(y, T_{0}\right) \frac{d y}{y} .
$$

Our choice will be $G(y)=K_{\mu}(y) y^{A}$ with $\mu$ purely imaginary and $A$ large positive. Then Lemma 13, Lemma 14, Lemma 15 and its Corollary together with (6) below will show that Theorem B is applicable for

$$
\varphi(x)-\left(\int_{-\infty}^{\infty} \widehat{\varphi}(t) \Gamma(1 / 4+i t) \Gamma(1 / 4-i t)(\operatorname{sh} \pi t) t d t\right) \varphi_{0}(x),
$$

where

$$
\varphi(x)=\int_{0}^{\infty} G(\pi|T| y) \varphi_{y}(x) \frac{d y}{y}
$$

and $\varphi_{0}$ is a fixed function (see Lemma 15 and its Corollary for the conditions satisfied by $\left.\varphi_{0}\right)$.

So applying Theorem B we get

$$
\begin{aligned}
I_{m, G}^{+}= & F_{0}+\frac{2 p_{N} s^{1 / 4}}{\sqrt{\pi}} \sum_{\substack{P Q=T_{0} \\
P>0,(N, P)=1}} \frac{1-i}{P}\left(\frac{D}{P}\right)|Q|^{1 / 2} \\
& \times\left\{\sum_{f_{j} \in V_{1 / 2}^{*}(4 N)} \frac{\bar{b}_{j, \infty}(s / D) b_{j, \infty}\left(D Q^{2}\right)}{\operatorname{ch}\left(\pi t_{j}\right)} \widehat{\varphi}\left(t_{j}\right)+\frac{E}{4 p_{N} \sqrt{s}|Q|}\right\}
\end{aligned}
$$

with

$$
\begin{aligned}
E= & \sum_{j=1}^{h} \int_{-\infty}^{\infty}\left(\frac{|D Q|^{2}}{s}\right)^{i t} \bar{\varphi}_{j, s / D, \infty}\left(\frac{1}{2}+i t\right) \\
& \times \varphi_{j, D Q^{2}, \infty / K}\left(\frac{1}{2}+i t\right) \frac{\widehat{\varphi}(t)}{\operatorname{ch}(\pi t)|\Gamma(3 / 4+i t)|^{2}} d t
\end{aligned}
$$

and

$$
F_{0}=f_{0} \int_{-\infty}^{\infty} \widehat{\varphi}(t) \Gamma(1 / 4+i t) \Gamma(1 / 4-i t)(\operatorname{sh} \pi t) t d t,
$$

where $f_{0}$ is a constant (depending on $\left.s, D, T_{0}, N\right)$.

We need to compute $\int_{0}^{\infty} J_{M}(x) \varphi_{y}(x) d x / x$. Use the substitution

$$
x=\pi|T| y\left(\sqrt{\frac{1}{\cos ^{2} \vartheta}+\frac{(R /(\pi T))^{2}}{y^{2}}}+\frac{\sin \vartheta}{\cos \vartheta}\right) .
$$


Then (3) is satisfied with $c=\pi|T| \sqrt{s} /(2 x), y=\operatorname{Im} z$. We have

$$
\frac{d x}{x}=\frac{d \vartheta}{\cos ^{2} \vartheta}\left(\frac{1}{\cos ^{2} \vartheta}+\frac{(R /(\pi T))^{2}}{y^{2}}\right)^{-1 / 2},
$$

so using (4), with the notations

(5) $\varphi_{M}(Y, \vartheta)$

$$
=\int_{-\infty}^{\infty} L_{M}\left(\sqrt{\frac{Y^{2}}{\cos ^{2} \vartheta}+R^{2}}+Y \frac{\sin \vartheta}{\cos \vartheta}\right)\left(\frac{Y^{2}}{\cos ^{2} \vartheta}+R^{2}\right)^{-1 / 2} e^{-i R} d R
$$

for $Y \in(0, \infty), \vartheta \in(-\pi / 2, \pi / 2)$, and $L_{M}(x)=J_{M}(x) / \sqrt{x}$, we obtain

$$
\int_{0}^{\infty} J_{M}(x) \varphi_{y}(x) \frac{d x}{x}=\sqrt{s}(\pi|T| y) \int_{-\pi / 2}^{\pi / 2} m\left(\frac{s}{n \cos ^{2} \vartheta}\right) \varphi_{M}(\pi|T| y, \vartheta) \frac{d \vartheta}{\cos ^{2} \vartheta}
$$

Since $\cos \vartheta$ is even, this gives

$$
\begin{aligned}
& \int_{0}^{\infty} J_{M}(x) \varphi(x) \frac{d x}{x} \\
& =\sqrt{s} \int_{-\pi / 2}^{\pi / 2} m\left(\frac{s}{n \cos ^{2} \vartheta}\right)\left(\int_{0}^{\infty} G(y) \frac{\varphi_{M}(y, \vartheta)+\varphi_{M}(y,-\vartheta)}{2} d y\right) \frac{d \vartheta}{\cos ^{2} \vartheta} .
\end{aligned}
$$

Now, Lemma 14 and the Corollary to Lemma 12 show that

$$
\widehat{\varphi}(t)=\sqrt{s} \int_{-\pi / 2}^{\pi / 2} m\left(\frac{s}{n \cos ^{2} \vartheta}\right) \Phi(t, \vartheta) \frac{d \vartheta}{\cos ^{2} \vartheta}
$$

where $\Phi(t, \vartheta)=O\left((1+|t|)^{-B}\right)$ with arbitrary positive $B$ for real $t$, uniformly in $\vartheta$ if $\vartheta$ is small; and we know $\Phi(t, 0)$ explicitly. Our idea now is to take $\vartheta=0$; this is possible, because an easy estimate shows

(8)

$$
\sum_{\left|t_{j}\right| \leq T} \frac{\left|b_{j, a}(n)\right|^{2}}{\operatorname{ch}\left(\pi t_{j}\right)}+\sum_{j=1}^{h} \int_{-T}^{T}\left|\varphi_{j n, a}\left(\frac{1}{2}+i t\right)\right|^{2} \frac{d t}{\operatorname{ch}(\pi t)|\Gamma(3 / 4+i t)|^{2}}=O\left(T^{B_{0}}\right)
$$

for any cusp $a$ of $\Gamma_{0}(4 N)$ for some $B_{0}$ (e.g. by using in formula (83) of [P] the function $\varphi$ of $[\mathrm{Du}], \S 5)$, where $b_{j, a}(n)$ is the $n$th Fourier coefficient of $f_{j}$ at $a$. So, if $m$ tends to the point $s / n$ in the sense that $m \geq 0$, its support shrinks to the point $s / n$, and always

$$
\int_{-\pi / 2}^{\pi / 2} m\left(\frac{s}{n \cos ^{2} \vartheta}\right) \frac{d \vartheta}{\cos ^{2} \vartheta}=1
$$


(we denote the process just described by $m \rightarrow s / n$ ), then we can substitute $\sqrt{s} \Phi\left(t_{j}, 0\right)$ and $\sqrt{s} \Phi(t, 0)$ in place of $\widehat{\varphi}\left(t_{j}\right)$ and $\widehat{\varphi}(t)$, respectively.

Now, by (6) and (7), and the definition of $\widehat{\varphi}$,

$\Phi(t, 0)=\beta(t) \int_{0}^{\infty} G(y)\left(\cos \pi(1 / 4+i t) \varphi_{2 i t}(y, 0)-\cos \pi(1 / 4-i t) \varphi_{-2 i t}(y, 0)\right) d y$

so by the Corollary to Lemma 12 ,

$$
\Phi(t, 0)=2 \sqrt{\pi} e(1 / 8) \operatorname{ch} \pi t \int_{0}^{\infty} G(y) y^{-1 / 2} K_{2 i t}(y) d y .
$$

We would like to take a fixed $t_{j}$ instead of the sum over $t_{j}$, and this explains our choice $G(y)=K_{\mu}(y) y^{A}$, in view of the relation

$$
\int_{0}^{\infty} K_{\mu}(x) K_{\nu}(x) x^{s-1} d x=2^{s-3} \Gamma(s)^{-1} \prod \Gamma\left(\frac{s \pm \mu \pm \nu}{2}\right)
$$

for $\operatorname{Re} s>|\operatorname{Re} \mu|+|\operatorname{Re} \nu|$ (see [I1], p. 228), which is a type of orthogonality relation with $s=0$.

So let $\tau \geq 0, \mu=i \tau, A$ a large positive number, and $G(y)=K_{\mu}(y) y^{A}$. In this case we have

$$
\Phi(t, 0)=2 \sqrt{\pi} e(1 / 8)(\operatorname{ch} \pi t) \Gamma(A, \tau, 2 t)
$$

with the notation

$$
\Gamma(A, \tau, t)=2^{A+1 / 2-3} \Gamma(A+1 / 2)^{-1} \prod \Gamma\left(\frac{A+1 / 2+i( \pm \tau \pm t)}{2}\right) .
$$

This proves that with $G(y)=K_{i \tau}(y) y^{A}$ we have

$$
\lim _{m \rightarrow s / n} I_{m, G}^{+}=I_{\tau}^{+}(A),
$$

where $I_{\tau}^{+}(A)$ is defined as the sum of $F_{0}(A)$ and

$$
\begin{aligned}
4 p_{N} \sqrt{2} s^{3 / 4} & \sum_{\substack{P Q=T_{0} \\
P>0,(N, P)=1}} \frac{|Q|^{1 / 2}}{P}\left(\frac{D}{P}\right) \\
& \times\left\{\sum_{f_{j} \in V_{1 / 2}^{*}(4 N)} \bar{b}_{j, \infty}\left(\frac{s}{D}\right) b_{j, \infty}\left(D Q^{2}\right) \Gamma\left(A, \tau, 2 t_{j}\right)+\frac{E(A)}{4 p_{N} \sqrt{s}|Q|}\right\},
\end{aligned}
$$

where

$$
\begin{aligned}
E(A)= & \sum_{j=1}^{h} \int_{-\infty}^{\infty}\left(\frac{|D Q|^{2}}{s}\right)^{i t} \bar{\varphi}_{j, s / D, \infty}\left(\frac{1}{2}+i t\right) \\
& \times \varphi_{j, D Q^{2}, \infty / K}\left(\frac{1}{2}+i t\right) \frac{\Gamma(A, \tau, 2 t)}{|\Gamma(3 / 4+i t)|^{2}} d t
\end{aligned}
$$




$$
F_{0}(A)=f_{1} \int_{-\infty}^{\infty} \Gamma(A, \tau, 2 t) \Gamma(1 / 4+i t) \Gamma(1 / 4-i t)(\operatorname{sh} 2 \pi t) t d t
$$

where $f_{1}$ is a constant (depending on $\left.s, D, T_{0}, N\right)$.

5. Inner product against cusp forms. Now we determine the inner product of $M(N, z)$ against a cusp form (of weight 0 ). Let $u$ be a cusp form for $\Gamma_{0}(N)$ with eigenvalue $\lambda$, and $d \mu(z)=y^{-2} d x d y$ be the invariant measure, and $F$ be a fundamental domain of $\Gamma_{0}(N)$ in $H$. Then

$$
\int_{F} M(N, z) u(z) d \mu(z)=\sum_{\gamma \in \Gamma_{N}} \omega_{D}(\gamma) \int_{F} m(z, \gamma z) u(z) d \mu(z) .
$$

We partition $\Gamma_{N}$ into conjugacy classes over $\Gamma_{0}(N)$ : for $\gamma \in \Gamma_{N}$ let $[\gamma]=$ $\left\{\tau^{-1} \gamma \tau: \tau \in \Gamma_{0}(N)\right\}$. We have $\tau_{1}^{-1} \gamma \tau_{1}=\tau_{2}^{-1} \gamma \tau_{2}$ if and only if $\tau_{2} \tau_{1}^{-1} \in$ $C(\gamma)=\left\{\sigma \in \Gamma_{0}(N): \gamma \sigma=\sigma \gamma\right\}$. So

$$
\begin{aligned}
T_{\gamma} & =\sum_{\delta \in[\gamma]} \omega_{D}(\delta) \int_{F} m(z, \delta z) u(z) d \mu(z) \\
& =\omega_{D}(\gamma) \sum_{\tau \in C(\gamma) \backslash \Gamma_{0}(N)} \int_{F} m\left(z, \tau^{-1} \gamma \tau z\right) u(z) d \mu(z) .
\end{aligned}
$$

Since $m\left(z, \tau^{-1} \gamma \tau z\right)=m(\tau z, \gamma \tau z)$ and $u(z)=u(\tau z)$, we finally obtain

$$
T_{\gamma}=\omega_{D}(\gamma) \int_{C(\gamma) \backslash H} m(z, \gamma z) u(z) d \mu(z),
$$

where $C(\gamma) \backslash H$ is a fundamental domain of $C(\gamma)$.

Now $\gamma$ is hyperbolic (since $s=t^{2}-4 n>0$ ). Denote its fixed points by $z_{1}$ and $z_{2}$. Then obviously $C(\gamma) \supseteq\left\{\sigma \in \Gamma_{0}(N): \sigma z_{1}=z_{1}, \sigma z_{2}=z_{2}\right\}$. Conversely, if $\sigma \gamma=\gamma \sigma$, then $\sigma$ permutes the set $\left\{z_{1}, z_{2}\right\}$; but $\sigma z_{1}=z_{2}$, $\sigma z_{2}=z_{1}$ is impossible, because then $\sigma^{2}=\mathrm{id}$, since it has at least three fixed points. This means that $\sigma$ is elliptic. But $\gamma$ cannot permute the fixed points of $\sigma$. So $C(\gamma)=\left\{\sigma \in \Gamma_{0}(N): \sigma z_{1}=z_{1}, \sigma z_{2}=z_{2}\right\}$.

It is easy to see that we may assume $c \neq 0$ in $\gamma=\left(\begin{array}{ll}a & b \\ c & d\end{array}\right)$, since in $[\gamma]$ there is always an element with lower left entry nonzero. Then the explicit form of the fixed points is $z_{1,2}=(a-d \pm \sqrt{s}) /(2 c)$. If $s$ is a square, then (denoting by $\widehat{\Gamma}_{0}(N)$ and $\widehat{C}(\gamma)$ the quotient by $\left\{\left(\begin{array}{ll}1 & 0 \\ 0 & 1\end{array}\right),\left(\begin{array}{cc}-1 & 0 \\ 0 & -1\end{array}\right)\right\}$ of $\Gamma_{0}(N)$ and $C(\gamma)$, respectively) $\widehat{C}(\gamma)=\{\mathrm{id}\}$, since id $\neq \sigma \in \widehat{\Gamma}_{0}(N)$ cannot have two rational fixed points. If $s$ is not a square, then $z_{1}$ and $z_{2}$ are quadratic irrational numbers, conjugate to each other over the rationals, so there is a id $\neq \sigma \in \widehat{\Gamma}_{0}(N)$ such that $\sigma$ has $z_{1}$ and $z_{2}$ as its fixed points (if $A, B$ and $C$ are integers with $A z_{1,2}^{2}+B z_{1,2}+C=0$ and $N \mid A, A \neq 0, D=B^{2}-4 A C$, then $D>0$ and $D$ is not a square, so there are positive integers $X, Y$ with 
$X^{2}-D Y^{2}=4$, and $\sigma=\left(\begin{array}{cc}(X-Y B) / 2 & -Y C \\ Y A & (X+Y B) / 2\end{array}\right)$ is suitable). Hence $\widehat{C}(\gamma)$ is infinite cyclic.

Let $h \in \mathrm{SL}(2, \mathbb{R})$ be such that $h^{-1} \gamma h z=R z(z \in H)$, where $R=$ $N(\gamma)>1$ is the norm of the hyperbolic transformation $\gamma$, i.e. $\sqrt{R}+1 / \sqrt{R}=$ $(a+d) / \sqrt{n}$, which means $R+1 / R-2=s / n$. In case $s$ is not a square, let $r_{0}>1$ be such that $\left(\begin{array}{cc}\sqrt{r_{0}} & 0 \\ 0 & 1 / \sqrt{r_{0}}\end{array}\right)$ is a generator of $h^{-1} \widehat{C}(\gamma) h$. We then have

$$
\begin{aligned}
T_{\gamma} & =\omega_{D}(\gamma) \int_{h^{-1}(C(\gamma) \backslash H)} m(h z, \gamma h z) u(h z) d \mu(z) \\
& =\omega_{D}(\gamma) \int_{h^{-1} C(\gamma) h \backslash H} m(z, R z) u(h z) d \mu(z),
\end{aligned}
$$

where we can take $h^{-1} C(\gamma) h \backslash H=\left\{z: 1 \leq|z|<r_{0}\right\}$ if $s$ is not a square, and $h^{-1} C(\gamma) h \backslash H=H$ otherwise. Let $I_{\gamma}=\left[1, r_{0}\right)$ if $s$ is not a square, and $I_{\gamma}=(0, \infty)$ otherwise. Since

$$
m(z, R z)=m\left(\frac{|R z-z|^{2}}{\operatorname{Im} z \operatorname{Im} R z}\right)
$$

with the substitution $z=r e^{i(\pi / 2+\vartheta)}$ we get

$$
\begin{aligned}
T_{\gamma} & =\int_{-\pi / 2}^{\pi / 2} \int_{I_{\gamma}} m\left(\frac{R+1 / R-2}{\cos ^{2} \vartheta}\right) u\left(h\left(r e^{i(\pi / 2+\vartheta)}\right)\right) \frac{r}{r^{2} \cos ^{2} \vartheta} d r d \vartheta \\
& =\int_{-\pi / 2}^{\pi / 2} m\left(\frac{s}{n \cos ^{2} \vartheta}\right) F\left(e^{i(\pi / 2+\vartheta)}\right) \frac{d \vartheta}{\cos ^{2} \vartheta}
\end{aligned}
$$

where

$$
F(z)=\int_{I_{\gamma}} u(h(r z)) \frac{d r}{r} \quad(z \in H) .
$$

Now, $F$ is constant on euclidean lines through the origin, i.e. $F(z)=$ $F(r z)$ for all $r>0$. If $I_{\gamma}=(0, \infty)$, then this is obvious, but it is also true for $I_{\gamma}=\left[1, r_{0}\right)$, because $u(h(z))$ is automorphic with respect to $h^{-1} \Gamma_{0}(N) h$, in particular $u\left(h\left(r_{0} z\right)\right)=u(h(z))$ for $z \in H$. So $F$ depends only on $\vartheta$ (if $\left.z=r e^{i(\pi / 2+\vartheta}\right)$, i.e. $F(z)=F(\vartheta)$, where $F$ is a function on $(-\pi / 2, \pi / 2)$.

On the other hand, since $u$ is an eigenfunction of the hyperbolic Laplace operator $\Delta$ with eigenvalue $\lambda$, so is $F(z)$. Using the form of the Laplace operator in polar coordinates:

$$
\Delta g=(r \cos \vartheta)^{2}\left(\frac{\partial^{2} g}{\partial r^{2}}+\frac{1}{r} \frac{\partial g}{\partial r}+\frac{1}{r^{2}} \frac{\partial^{2} g}{\partial \vartheta^{2}}\right)
$$

for $g=g(r, \vartheta)$, we find that $F(\vartheta)$ satisfies a second order ordinary differential 
equation, which depends only on $\lambda$ :

$$
F^{(2)}(\vartheta)=\frac{\lambda}{\cos ^{2} \vartheta} F(\vartheta) \quad(\vartheta \in(-\pi / 2, \pi / 2)) .
$$

Let $f_{\lambda}(\vartheta)$ be the solution of this differential equation with $f_{\lambda}(0)=1$, $f_{\lambda}^{(1)}(0)=0$, and $\widetilde{f}_{\lambda}(\vartheta)$ the one with $\widetilde{f}_{\lambda}(0)=0, \widetilde{f}_{\lambda}^{(1)}(0)=1$. Then $F(\vartheta)=$ $F(0) f_{\lambda}(\vartheta)+F^{(1)}(0) \widetilde{f}_{\lambda}(\vartheta)$, and $\widetilde{f}_{\lambda}(\vartheta)$ is an odd function, so it gives 0 in $T_{\gamma}$, i.e.

$$
T_{\gamma}=F(0) \int_{-\pi / 2}^{\pi / 2} m\left(\frac{s}{n \cos ^{2} \vartheta}\right) f_{\lambda}(\vartheta) \frac{d \vartheta}{\cos ^{2} \vartheta} .
$$

Here

$$
F(0)=\int_{I_{\gamma}} u(h(r i)) \frac{d r}{r}=\int_{C_{\gamma}} u d S,
$$

where $d S=|d z| / y$ is the hyperbolic arc length, and $C_{\gamma}$ is the closed geodesic $C(\gamma) \backslash l_{\gamma}$, where $l_{\gamma}$ is the noneuclidean line connecting the fixed points $\left(z_{1}\right.$ and $z_{2}$ ) of $\gamma$.

We now describe the conjugacy classes of $\Gamma_{N}=\Gamma_{n, t, N}$ over $\Gamma_{0}(N)$. If $\gamma=\left(\begin{array}{ll}a & b \\ c & d\end{array}\right)$, let

$$
Q_{\gamma}(X, Y)=(-Y X)\left(\begin{array}{ll}
a & b \\
c & d
\end{array}\right)\left(\begin{array}{l}
X \\
Y
\end{array}\right)=c X^{2}+(d-a) X Y-b Y^{2} .
$$

It is easy to see that the correspondence $\gamma \leftrightarrow Q_{\gamma}$ is one-to-one between $\Gamma_{n, t, N}$ and $Q_{N, s}$. If $\left(\begin{array}{c}\tilde{X} \\ \tilde{Y}\end{array}\right)=\tau\left(\begin{array}{c}X \\ Y\end{array}\right)$ with some $\tau \in \Gamma_{0}(N)$, then $(-\tilde{Y} \tilde{X})=$ $(-Y X) \tau^{-1}$ (since $\operatorname{det} \tau=1$ ), and it follows that if $\gamma_{1}, \gamma_{2} \in \Gamma_{n, t, N}$, then $\gamma_{1}$ and $\gamma_{2}$ are conjugate over $\Gamma_{0}(N)$ (i.e. $\gamma_{2}=\delta^{-1} \gamma_{1} \delta$ with some $\delta \in \Gamma_{0}(N)$ ) if and only if $Q_{\gamma_{1}}$ and $Q_{\gamma_{2}}$ are equivalent over $\Gamma_{0}(N)$. From these considerations we see that $\gamma \leftrightarrow Q_{\gamma}$ defines a one-to-one correspondence between the conjugacy classes of $\Gamma_{n, t, N}$ over $\Gamma_{0}(N)$, and $\Lambda_{N, s}$. If $Q \in Q_{N, s}$, then $C_{Q, N}=C_{\gamma}$ if $\gamma$ is the element of $\Gamma_{n, t, N}$ with $Q=Q_{\gamma}$, so we have proved the following lemma.

Lemma 2. If $u$ is a cusp form on $\Gamma_{0}(N)$ with eigenvalue $\lambda$, then

$$
\begin{aligned}
& \int_{F} M(N, z) u(z) d \mu(z) \\
& \quad=\left(\sum_{Q \in \Lambda_{N, s}} \omega_{D}(Q) \int_{C_{Q, N}} u d S\right)\left(\int_{-\pi / 2}^{\pi / 2} m\left(\frac{s}{n \cos ^{2} \vartheta}\right) f_{\lambda}(\vartheta) \frac{d \vartheta}{\cos ^{2} \vartheta}\right) .
\end{aligned}
$$

We would like to apply the spectral theorem for $M(N, z)$, but for this we have to determine its behaviour near cusps, and subtract from $M(N, z)$ its main terms near the cusps. 
6. The behaviour of $M(N, z)$ near cusps. In this section $N$ will be fixed, so in general we do not indicate the dependence on $N$, writing e.g. $M(z)=M(N, z), M_{0}(z)=M_{0}(N, z)$. Let $F$ be a fundamental domain of $\Gamma_{0}(N)$, and

$$
P(Y)=\{z=x+i y: 0<x \leq 1, y>Y\} .
$$

If $Y_{0}$ is large enough, the cuspidal zones $F_{q}\left(Y_{0}\right)=\sigma_{q} P\left(Y_{0}\right)$ are disjoint ( $q$ runs over a complete set of $\Gamma_{0}(N)$-inequivalent cusps), and the fundamental domain $F$ is partitioned into

$$
F=F\left(Y_{0}\right) \cup \bigcup_{q} F_{q}\left(Y_{0}\right)
$$

where $F\left(Y_{0}\right)$ is the central part,

$$
F\left(Y_{0}\right)=F \backslash \bigcup_{q} F_{q}\left(Y_{0}\right),
$$

and $F\left(Y_{0}\right)$ has compact closure.

Let $q=u / v$ be a cusp of $\Gamma_{0}(N)$, where $v>0, v \mid N,(u, v)=1$ (every cusp is $\Gamma_{0}(N)$-equivalent to a cusp of this form). Then it is not difficult to check (see [I2]) that the "scaling matrix" of $q$ is $\sigma_{q}=\tau_{q} \varrho_{q}$ with

$$
\tau_{q}=\left(\begin{array}{ll}
u & x \\
v & y
\end{array}\right) \in \mathrm{SL}(2, \mathbb{Z}) \text { and } \varrho_{q}=\left(\begin{array}{cc}
\sqrt{m_{q}} & 0 \\
0 & 1 / \sqrt{m_{q}}
\end{array}\right),
$$

where $m_{q}=N /\left(N, v^{2}\right)$. This means that $\sigma_{q} \infty=q$ and $\sigma_{q}^{-1} \gamma_{q} \sigma_{q}=\left(\begin{array}{ll}1 & 1 \\ & 1\end{array}\right)$, where $\gamma_{q}$ is a generator of the stability group of $q$ in $\Gamma_{0}(N)$ (we denote this stability group by $\Gamma_{q}$ ). We use these notations in the following lemmas.

We now describe the behaviour of $M(z)$ near cusps. Our function $m$ is compactly supported on $(0, \infty)$; it will vary, and its support will shrink to $s / n$, so we assume in the sequel e.g. that $m(x)=0$ for $x>2 s / n$, i.e. the support is universally bounded.

LEMMA 3. There is a constant $Y_{1}$ such that if $q=u / v$ is a cusp of $\Gamma_{0}(N)$, then for $z \in F_{q}\left(Y_{1}\right)$ we have $M(z)=M_{0}^{q}(z)$, where

$$
M_{0}^{q}(z)=\sum_{\gamma \in \Gamma_{N}, \gamma q=q} \omega_{D}(\gamma) m(z, \gamma z) .
$$

Proof. It is enough to prove that if $z \in P\left(Y_{1}\right)$, then $m\left(\sigma_{q} z, \gamma \sigma_{q} z\right)=$ $m\left(z, \sigma_{q}^{-1} \gamma \sigma_{q} z\right)=0$ for $\gamma \in \Gamma_{N}, \gamma q \neq q$, i.e. that $|z-\delta z|^{2} /(\operatorname{Im} z \operatorname{Im} \delta z)>2 s / n$ with the notation $\delta=\sigma_{q}^{-1} \gamma \sigma_{q}$ if $\delta \infty \neq \infty$. But this is true if $Y_{1}$ is large enough, because $\operatorname{Im} z \geq Y_{1}, \operatorname{Im} \delta z=n \operatorname{Im} z /|c z+d|^{2} \leq n /\left(c^{2} \operatorname{Im} z\right)$, where $c$ is the lower left entry of $\delta$, and $c^{2}$ is bounded below if it is nonzero. 
Next we describe the Fourier expansion of the function $M_{0}^{q}(z)$ at $q$ (obviously $\left.M_{0}^{q}\left(\gamma_{q} z\right)=M_{0}^{q}(z)\right)$. For this let

$$
M_{0}^{q}(y, k)=\int_{0}^{1} M_{0}^{q}\left(\sigma_{q}(\xi+i y)\right) e(k \xi) d \xi .
$$

The sum defining $M_{0}^{q}(z)$ is empty if $s$ is not a square, because then the fixed points of any $\gamma \in \Gamma_{N}$ are irrational.

Lemma 4. Let $s$ be a square and $k$ be an integer. Then

$$
M_{0}^{q}(y, k)=2 c_{q}(k, s) y m_{F}(|k| y),
$$

where

$$
\begin{aligned}
c_{q}(k, s) & =\sum_{\substack{\beta(\bmod \sqrt{s}) \\
\beta m_{q} \equiv 0(\bmod 1), N \mid y v \sqrt{s}-v^{2} \beta m_{q}}} \omega_{D}\left(\sqrt{s} X Y+\beta m_{q} Y^{2}\right) e\left(-\frac{k \beta}{\sqrt{s}}\right), \\
m_{F}(\alpha) & =\int_{-\infty}^{\infty} m\left(\frac{s}{n}\left(1+\xi^{2}\right)\right) e(\alpha \xi) d \xi .
\end{aligned}
$$

The statement is also true if $s$ is not a square, with $c_{q}(k, s)=0$.

Proof. We have $M_{0}^{q}\left(\sigma_{q} z\right)=\sum_{\gamma \in \Gamma_{N}, \gamma q=q} \omega_{D}(\gamma) m\left(z, \sigma_{q}^{-1} \gamma \sigma_{q} z\right)$. Here $\delta \infty=\infty$ for $\delta=\sigma_{q}^{-1} \gamma \sigma_{q}$, so $\delta=\left(\begin{array}{cc}a & b \\ d\end{array}\right)$, where $a d=n, a+d=t$. Then $a-d= \pm \sqrt{s}, a$ and $d$ are integers, $\varrho_{q} \delta \varrho_{q}^{-1}=\left(\begin{array}{c}a b m_{q} \\ d\end{array}\right)$, and the lower left entry of $\gamma=\sigma_{q} \delta \sigma_{q}^{-1}$ is $y v(a-d)-v^{2} b m_{q}$. So the condition $\gamma \in \Gamma_{0}(N)$ means that $b m_{q}$ is an integer, and that $N$ divides the lower left entry of $\gamma$. These conditions depend only on $b(\bmod 1)$ (because $N$ divides $\left.v^{2} m_{q}\right)$, and we see that $a-d=+\sqrt{s}$ and $a-d=-\sqrt{s}$ give the same sum; we take the plus sign. It follows easily from the definition (and from $s / D \equiv 0,1(\bmod 4))$ that $\omega_{D}(\gamma)=\omega_{D}\left(\sqrt{s} X Y+b m_{q} Y^{2}\right)$ depends only on $b(\bmod \sqrt{s})$. This shows that for $z=\xi+i y$, since $|\sqrt{s} z+b|^{2}=(\sqrt{s} \xi+b)^{2}+s y^{2}$, we get

$$
\begin{aligned}
M_{0}^{q}(z)=2 & \sum_{\substack{\beta(\bmod \sqrt{s}) \\
\beta m_{q} \equiv 0(\bmod 1), N \mid y v \sqrt{s}-v^{2} \beta m_{q}}} \omega_{D}\left(\sqrt{s} X Y+\beta m_{q} Y^{2}\right) \\
& \times \sum_{b \equiv \beta(\bmod \sqrt{s})} m\left(\frac{s}{n}\left(1+\left(\frac{\xi+b / \sqrt{s}}{y}\right)^{2}\right)\right) .
\end{aligned}
$$

(Note that this sum is empty if $(N / v, v)$ does not divide $\sqrt{s}$.) So $M_{0}^{q}(y, k)$ is 2 times the sum over the same set of $\beta$ of

$$
\int_{-\infty}^{\infty} m\left(\frac{s}{n}\left(1+\left(\frac{\xi+\beta / \sqrt{s}}{y}\right)^{2}\right)\right) e(k \xi) d \xi
$$


which implies the lemma by the substitution $y^{-1}(\xi+\beta / \sqrt{s}) \rightarrow \xi$, because the function $\mu$ is even.

The last statement is obvious by the remark preceding the lemma.

Corollary. Let $G \in L_{1}((0, \infty)), T_{0} \neq 0$ an integer, $T=2 T_{0}$. Then with a constant $c\left(N, T_{0}, s\right)$ we have

$$
\lim _{m \rightarrow s / n} \int_{0}^{\infty} G(|\pi T| y)\left(\int_{0}^{1} M_{0}(N, \xi+i y) e\left(T_{0} \xi\right) d \xi\right) \frac{d y}{y}=c\left(N, T_{0}, s\right) \int_{0}^{\infty} G(y) d y .
$$

(The meaning of $m \rightarrow s / n$ is given after (8), and $M_{0}(N, z)$ is given in (2).)

Pr o of. Since $\infty$ and $1 / N$ are equivalent over $\Gamma_{0}(N)$, we have

$$
\int_{0}^{1} M_{0}(\xi+i y) e\left(T_{0} \xi\right) d \xi=2 c_{1 / N}\left(T_{0}, s\right) y m_{F}\left(\left|T_{0}\right| y\right) .
$$

Because $T=2 T_{0}$, we need the limit of

$$
\frac{c_{1 / N}\left(T_{0}, s\right)}{\pi\left|T_{0}\right|} \int_{0}^{\infty} G(y) m_{F}\left(\frac{y}{2 \pi}\right) d y \quad \text { as } m \rightarrow s / n .
$$

If $m \rightarrow s / n$, then $m_{F}(y /(2 \pi)) \rightarrow 1$ for any given $y$, and always $\left|m_{F}(y /(2 \pi))\right|$ $\leq 1$, which implies the assertion.

We now subtract from $M(z)$ the main terms near the cusps. Assume in the sequel that

$$
m_{F}(0)=\int_{-\pi / 2}^{\pi / 2} m\left(\frac{s}{n \cos ^{2} \vartheta}\right) \frac{d \vartheta}{\cos ^{2} \vartheta}=1
$$

Let $0<Y_{0}<Y_{1}$, where $Y_{0}$ is as in the description of the fundamental domain $F$ (see (12)), and $Y_{1}$ is as in Lemma 3, i.e. for any $q$, if $z \in F_{q}\left(Y_{1}\right)$, we have $M(z)=M_{0}^{q}(z)$. Furthermore let $\psi$ be a smooth real-valued function on $(0, \infty)$ with

Define

$$
\psi(y)= \begin{cases}y & \text { for } y \geq Y_{1} \\ 0 & \text { for } y \leq Y_{0}\end{cases}
$$

$$
M_{\psi}(N, z)=M_{\psi}(z)=M(z)-\sum_{q} 2 c_{q}(0, s) E_{q}(z \mid \psi),
$$

where $E_{q}(z \mid \psi)$ is the weighted Eisenstein series

$$
E_{q}(z \mid \psi)=\sum_{\gamma \in \Gamma_{q} \backslash \Gamma_{0}(N)} \psi\left(\operatorname{Im} \sigma_{q}^{-1} \gamma z\right) .
$$

Observe that $M(z)-M_{\psi}(z)$ does not depend on $m$. 
It follows easily from the conditions on $Y_{0}$ and $\psi$ that if $z \in F_{q_{1}}\left(Y_{0}\right)$, then $E_{q_{2}}(z \mid \psi)=\delta_{q_{1} q_{2}} \psi\left(\operatorname{Im} \sigma_{q_{1}}^{-1} z\right)$. So, if $z \in F_{q}\left(Y_{1}\right)$, by Lemmas 3 and 4 we have

$$
M_{\psi}(z)=M_{0}^{q}(z)-M_{0}^{q}\left(\operatorname{Im} \sigma_{q}^{-1} z, 0\right),
$$

since $m_{F}(0)=1$. Hence, if $z \in P\left(Y_{1}\right)$ and $z=\xi+i y$, Lemma 4 implies $(m$ is smooth)

$$
M_{\psi}\left(\sigma_{q} z\right)=2 y \sum_{k \neq 0} c_{q}(-k, s) m_{F}(|k| y) e(k \xi) .
$$

We shall need a very crude bound for the inner product of $M_{\psi}(z)$ against an Eisenstein series (of weight 0).

Lemma 5. Let $m \rightarrow s / n$. There is a function $H(t)$ on $(-\infty, \infty)$ which is bounded on every finite interval,

$$
\int_{-\infty}^{\infty} \frac{H^{2}(t)}{(1+|t|)^{20}} d t<\infty
$$

and

$$
\left|\int_{F} M_{\psi}(z) E_{r}\left(z, \frac{1}{2}+i t\right) \frac{d x d y}{y^{2}}\right| \leq H(t)
$$

if $r$ is a cusp of $\Gamma_{0}(N), t$ is a real number, and $E_{r}(z, 1 / 2+i t)$ is the corresponding Eisenstein series of $\Gamma_{0}(N)$. It is important that $H(t)$ does not depend on $m$.

Proof. At another cusp $q$ the Eisenstein series $E_{r}(z, 1 / 2+i t)$ has the Fourier expansion

$$
\begin{aligned}
& E_{r}\left(\sigma_{q} z, 1 / 2+i t\right) \\
& \quad=\delta_{q r} y^{1 / 2+i t}+\varphi_{q r}(1 / 2+i t) y^{1 / 2-i t}+\sum_{k \neq 0} \varphi_{q r}(k, 1 / 2+i t) W_{1 / 2+i t}(k z) .
\end{aligned}
$$

The integral in question converges absolutely, because the decay of $m_{F}$ is fast. On $F_{q}\left(Y_{1}\right)$ the integral is

$$
2 \sum_{k \neq 0} c_{q}(k, s) \varphi_{q r}(k, 1 / 2+i t) \int_{Y_{1}}^{\infty} m_{F}(|k| y) W_{1 / 2+i t}(i k y) \frac{d y}{y} .
$$

Since always $\left|m_{F}\right| \leq 1$ and $c_{q}(k, s)$ is bounded (depending only on $s$ and $N$ ), this can be estimated by

$$
\sum_{k \neq 0}\left|\varphi_{q r}(k, 1 / 2+i t)\right| \int_{2 \pi|k| Y_{1}}^{\infty}\left|K_{i t}(y)\right| \frac{d y}{y^{1 / 2}},
$$


and by (9), and formula (5.10.24) of [Le], there are positive constants $c_{1}$ and $c_{2}$ such that it is of order

$$
\begin{aligned}
H_{1, q}(t)= & \left(1+|t|^{1 / 2}\right) e^{-(\pi / 2)|t|}\left(\sum_{\substack{k \neq 0 \\
|k| \leq c_{1}(1+|t|)}}\left|\varphi_{q r}(k, 1 / 2+i t)\right|\right. \\
& \left.+\sum_{\substack{k \neq 0 \\
|k| \geq c_{1}(1+|t|)}} e^{-c_{2}|k|}\left|\varphi_{q r}(k, 1 / 2+i t)\right|\right) .
\end{aligned}
$$

This is obviously bounded on every finite interval, and by formulas (8.27) and (8.6) of [I1] we have $\int_{-\infty}^{\infty}\left(H_{1, q}^{2}(t) /(1+|t|)^{20}\right) d t<\infty$.

It remains to estimate the integral on $F\left(Y_{1}\right)$. Since $F\left(Y_{1}\right)$ has compact closure, there are only a finite number of $\gamma \in \Gamma_{N}$ for which $|z-\gamma z|^{2} /(\operatorname{Im} z \operatorname{Im} \gamma z) \leq 2 s / n$ with some $z \in F\left(Y_{1}\right)$, and it is easy to see that for a fixed $\gamma$ the integral $\int_{F\left(Y_{1}\right)} m(z, \gamma z) d x d y / y^{2}$ is bounded as $m \rightarrow s / n$. Since $M(z)-M_{\psi}(z)$ is fixed, the $F\left(Y_{1}\right)$-part of our integral can be estimated by the maximum of $\left|E_{r}(z, 1 / 2+i t)\right|$ on the closure of $F\left(Y_{1}\right)$, and our very weak estimate follows e.g. by estimating trivially the Fourier expansion of the Eisenstein series, and by (8.24) of [I1].

We shall several times use the following analytic lemma.

Lemma 6. Let $r(t)$ be a function on $(-\infty, \infty)$ such that

$$
\lim _{|t| \rightarrow \infty}|r(t)| e^{-(\pi / 2)|t|}(1+|t|)^{B}=0
$$

for any positive $B$, and let $R(t)$ be a given function on $(-\infty, \infty)$ which is bounded on every finite interval,

$$
|r(t)| \leq e^{(\pi / 2)|t|} R(t)
$$

and e.g.

$$
\int_{-\infty}^{\infty} \frac{R^{2}(t)}{(1+|t|)^{40}} d t<\infty
$$

and let $\tau \geq 0$. Define

$$
F_{\tau, r}(\alpha)=\int_{0}^{\infty} K_{i \tau}(y) y^{\alpha}\left(\int_{-\infty}^{\infty} r(t) K_{i t}(y) d t\right) \frac{d y}{y}
$$

for $\operatorname{Re} \alpha>0$. Then $F_{\tau, r}$ is a regular function, and on any compact subset of $\{\operatorname{Re} \alpha>0\},\left|F_{\tau, r}(\alpha)\right|$ is smaller than a constant depending only on $\tau, R$ and this subset. Furthermore, $(\alpha+\tau)^{2}\left|F_{\tau, r}(\alpha)\right|$ is smaller than a constant depending only on $\tau$ and $R$ if $0<\alpha<1$ (i.e. for $\tau>0$ we take $\left|F_{\tau, r}(\alpha)\right|$, for $\tau=0$ we take $\left.\alpha^{2}\left|F_{\tau, r}(\alpha)\right|\right)$. 
Proof. It is clear that the double integral is absolutely convergent, and changing the order of integration we get, by (9),

$$
F_{\tau, r}(\alpha)=2^{\alpha-3} \frac{1}{\Gamma(\alpha)} \int_{-\infty}^{\infty} r(t) \prod \Gamma\left(\frac{\alpha+i( \pm t \pm \tau)}{2}\right) d t .
$$

Here we estimate $r$ by $R$, and use Stirling's formula and elementary estimates to get the result. (The point is that the product of $\Gamma$-functions decays much faster as $|t| \rightarrow \infty$ than $K_{i t}(y)$ for a fixed $y$, so we can use for $r$ the weaker but universal bound, while the stronger (but not universal) bound is needed for the absolute convergence of the double integral.)

We will handle $M(z)-M_{\psi}(z)$ in the following lemma.

Lemma 7. Let $T_{0} \neq 0$ be an integer,

$$
E_{q, \psi}\left(y, T_{0}\right)=\int_{0}^{1} E_{q}(\xi+i y \mid \psi) e\left(T_{0} \xi\right) d \xi
$$

$T=2 T_{0}, \tau \geq 0$, and

$$
Y(A)=\int_{0}^{\infty} K_{i \tau}(|\pi T| y)(|\pi T| y)^{A} E_{q, \psi}\left(y, T_{0}\right) \frac{d y}{y} .
$$

Then $Y(A)$ is absolutely convergent for $\operatorname{Re} A>0$, it extends meromorphically to $\operatorname{Re} A>-1 / 2$, it may have singularities only at $A= \pm i \tau$, and $(A+1 / 2+\tau)^{2}|Y(A)|$ is bounded as $A \rightarrow-1 / 2+0$ (i.e. $A$ tends to $-1 / 2$ on the real axis).

Proof. By (3.17) of [I1], for $y>0$ we have

$$
E_{q, \psi}\left(y, T_{0}\right)=\sum_{c>0} S_{q \infty}\left(0,-T_{0} ; c\right) \int_{-\infty}^{\infty} \psi\left(\frac{y c^{-2}}{t^{2}+y^{2}}\right) e\left(T_{0} t\right) d t .
$$

We use the formula (see [I1], (3.19))

$$
\int_{-\infty}^{\infty}\left(t^{2}+y^{2}\right)^{-s} e\left(T_{0} t\right) d t=2 \frac{\pi^{s}}{\Gamma(s)}\left(\frac{\left|T_{0}\right|}{y}\right)^{s-1 / 2} K_{s-1 / 2}\left(2 \pi\left|T_{0}\right| y\right),
$$

write $\psi(Y)=Y+(\psi(Y)-Y)$, and with some $1 / 2<\sigma=\operatorname{Re} s<1$,

$$
\psi(Y)-Y=\frac{1}{2 \pi i} \int_{(\sigma)} \psi^{*}(s) Y^{s} d s, \quad \psi^{*}(s)=\int_{0}^{\infty}(\psi(Y)-Y) Y^{-s-1} d Y .
$$

This gives

$$
\begin{aligned}
E_{q, \psi}\left(y, T_{0}\right)= & 2\left(\left|T_{0}\right| y\right)^{1 / 2} \varphi_{q \infty}\left(-T_{0}, 1\right) K_{1 / 2}\left(2 \pi\left|T_{0}\right| y\right) \\
& +\frac{1}{\pi i}\left(\left|T_{0}\right| y\right)^{1 / 2} \int_{(\sigma)} \varphi_{q \infty}\left(-T_{0}, s\right) K_{s-1 / 2}\left(2 \pi\left|T_{0}\right| y\right) \psi^{*}(s) d s,
\end{aligned}
$$


where

$$
\varphi_{q \infty}\left(-T_{0}, s\right)=\frac{\pi^{s}}{\Gamma(s)}\left|T_{0}\right|^{s-1}\left(\sum_{c>0} \frac{S_{q \infty}\left(0,-T_{0} ; c\right)}{c^{2 s}}\right) .
$$

This is justified by the facts that on a line $\sigma=\sigma_{0}$, where $\sigma_{0}<1$, the function $\psi^{*}(s)$ decays faster than any power of $|\operatorname{Im} s|$ (see the conditions on $\psi$ ), and $S_{q \infty}\left(0,-T_{0} ; c\right)$ is bounded in $c$, so the Dirichlet series is absolutely convergent for $\sigma>1 / 2$. We can move the line of integration to $\sigma=1 / 2$ because by the general estimate (8.27) of [I1], $\left|\varphi_{q \infty}\left(-T_{0}, 1 / 2+i t\right)\right|$ is bounded on average by $e^{(\pi / 2)|t|}|t|^{1 / 2}$, and the decay of $\psi^{*}(s)$ is fast on this line as well. Now (remember $T=2 T_{0}$ ) the lemma follows from these estimates, from Lemma 6 , and because the $K_{1 / 2}$-part of $E_{q, \psi}\left(y, T_{0}\right)$ gives in $Y(A)$ a constant times

$$
2^{A} \Gamma(A+1 / 2)^{-1} \prod \Gamma\left(\frac{1 / 2+A \pm 1 / 2 \pm i \tau}{2}\right) .
$$

7. Proof of the basic identity. Our aim in this section is to prove Lemma 10 below. Theorems 1 and 2 will be easy consequences of that lemma.

Let $U_{N}$ be a complete orthonormal system of Maass cusp forms of weight 0 for $\Gamma_{0}(N)$ (we do not index them in the usual way to avoid confusion because of the presence of weight 0 and weight $1 / 2$ Maass forms). For $u \in U_{N}$ we denote by $\lambda_{u}$ its Laplace eigenvalue, i.e. $\Delta u=\lambda_{u} u, s_{u}\left(s_{u}-1\right)=\lambda_{u}$, $s_{u}=1 / 2+i t_{u}, t_{u} \geq 0$ or $i t_{u} \geq 0$, and let its Fourier expansion be

$$
u(z)=\sum_{k \neq 0} a_{u}(k) W_{s_{u}}(k z) .
$$

Lemma 8. Let $\tau \geq 0$ be fixed, $T_{0} \neq 0$ an integer, $T=2 T_{0}$, and

$$
X_{m}(A)=\int_{0}^{\infty} K_{i \tau}(|\pi T| y)(|\pi T| y)^{A}\left(\int_{0}^{1} M_{\psi}(\xi+i y) e\left(T_{0} \xi\right) d \xi\right) \frac{d y}{y} .
$$

Then $X_{m}(A)$ is meromorphic for $\operatorname{Re} A>-1 / 2$. Denote by $X_{m}^{*}(A)$ the difference of $X_{m}(A)$ and

$$
\begin{aligned}
\left(\frac{2}{\pi}\right)^{1 / 2} \sum_{u \in U_{N}^{\tau,+}} & \left(\sum_{Q \in \Lambda_{N, s}} \omega_{D}(Q) \int_{C_{Q, N}} \bar{u} d S\right) \\
& \times\left(\int_{-\pi / 2}^{\pi / 2} m\left(\frac{s}{n \cos ^{2} \vartheta}\right) f_{\lambda_{u}}(\vartheta) \frac{d \vartheta}{\cos ^{2} \vartheta}\right) a_{u}\left(-T_{0}\right) \Gamma\left(A, \tau, t_{u}\right),
\end{aligned}
$$

where $U_{N}^{\tau,+}=\left\{u \in U: t_{u}=\tau\right.$ or $\left.i t_{u}>0\right\}$. Then $X_{m}^{*}(A)$ is regular for $\operatorname{Re} A>-1 / 2$, and in any compact subset of $\{\operatorname{Re} A>-1 / 2\},\left|X_{m}^{*}(A)\right|$ is smaller than a constant depending on the subset but independent of $m$; fur- 
thermore $(A+1 / 2+\tau)^{2}\left|X_{m}^{*}(A)\right|$ is bounded by a constant independent of $m$ if $-1 / 2<A<1 / 2$.

Proof. The spectral theorem is obviously applicable for $M_{\psi}(z)$ in view of the Fourier expansion (13). Since $M(z)-M_{\psi}(z)$ is a linear combination of weighted Eisenstein series, it is orthogonal to cusp forms, so by Lemmas 2 and 5 and using the Fourier expansion of cusp forms and Eisenstein series ( $m, \psi$ and the functions $f_{\lambda}$ are real) we see that $\int_{0}^{1} M_{\psi}(\xi+i y) e\left(T_{0} \xi\right) d \xi$ is the sum of

$$
\begin{array}{r}
2\left(\left|T_{0}\right| y\right)^{1 / 2} \sum_{u \in U_{N}}\left(\sum_{Q \in \Lambda_{N, s}} \omega_{D}(Q) \int_{C_{Q, N}} \bar{u} d S\right)\left(\int_{-\pi / 2}^{\pi / 2} m\left(\frac{s}{n \cos ^{2} \vartheta}\right) f_{\lambda_{u}}(\vartheta) \frac{d \vartheta}{\cos ^{2} \vartheta}\right) \\
\times a_{u}\left(-T_{0}\right) K_{i t_{u}}\left(2 \pi\left|T_{0}\right| y\right)
\end{array}
$$

and

$$
2\left(\left|T_{0}\right| y\right)^{1 / 2} \frac{1}{4 \pi} \sum_{r} \int_{-\infty}^{\infty} \chi_{m, r}(t) \varphi_{r \infty}\left(-T_{0}, 1 / 2+i t\right) K_{i t}\left(2 \pi\left|T_{0}\right| y\right) d t,
$$

where $\left|\chi_{m, r}(t)\right| \leq H(-t)$ (see Lemma 5). Now, since $T=2 T_{0}$, the lemma follows by (8.27) and (8.6) of [I1], by our Lemma 6, (9) and (10), elementary estimates, and by the fact that always $\left|f_{\lambda}(\vartheta)\right| \leq 1$, which follows easily from the shape of the differential equation satisfied by $f_{\lambda}$.

Let $\tau \geq 0$ be fixed, $G(y)=K_{i \tau}(y) y^{A}$, define

$$
M^{*}(z)=\sum_{L \mid N} \frac{\left(\frac{D}{L}\right)}{L} \mu(L) M(N / L, L z),
$$

and

$$
I_{m, \tau}(A)=\int_{0}^{\infty} G(|\pi T| y)\left(\int_{0}^{1} M^{*}(\xi+i y) e\left(T_{0} \xi\right) d \xi\right) \frac{d y}{y} .
$$

The following identity is obvious.

Lemma 9. If $F$ is any function on $H$ which is periodic with respect to 1 , $L>0$ is an integer and $T_{0}$ is an integer, $T=2 T_{0}$, then, assuming absolute convergence,

$$
\begin{aligned}
\int_{0}^{\infty} G(|\pi T| y)\left(\int_{0}^{1} F(L(\xi+i y)) e\left(T_{0} \xi\right) d \xi\right) \frac{d y}{y} \\
=\int_{0}^{\infty} G\left(\left|\pi \frac{T}{L}\right| y\right)\left(\int_{0}^{1} F(\xi+i y) e\left(\frac{T_{0}}{L} \xi\right) d \xi\right) \frac{d y}{y}
\end{aligned}
$$

for $L \mid T_{0}$, and the left-hand side is 0 otherwise. 
This, (1), the Corollary to Lemma 4, and (11) show that if we define (remember $G(y)=K_{i \tau}(y) y^{A}$ )

$$
I_{\tau}(A)=2 I_{\tau}^{+}(A)+c^{*}\left(N, T_{0}, s\right) \int_{0}^{\infty} G(y) d y
$$

with a suitable constant $c^{*}\left(N, T_{0}, s\right)$, then, if $A$ is a large enough positive number,

$$
\lim _{m \rightarrow s / n} I_{m, \tau}(A)=I_{\tau}(A) .
$$

On the other hand, we know (Lemmas 7-9) that the functions $I_{m, \tau}(A)$ and $I_{\tau}(A)$ are meromorphic for $\operatorname{Re} A>-1 / 2$, and there is a finite subset $S=S_{\tau, N, T_{0}}$ of $\{\operatorname{Re} A>-1 / 2\}$ such that on $\{\operatorname{Re} A>-1 / 2\} \backslash S$ each function is regular, and on every compact subset of this domain each function is smaller than a bound depending on the subset but independent of $m$. This implies that $\lim _{m \rightarrow s / n} I_{m, \tau}(A)=I_{\tau}(A)$ for every $A \in\{\operatorname{Re} A>-1 / 2\} \backslash S$ (we use the following function-theoretic result: if $f_{1}, f_{2}, \ldots$ and $f$ are regular functions on a domain, if there is a constant $C$ such that $\left|f_{n}\right|,|f| \leq C$ for every $n$, and if $\lim _{n \rightarrow \infty} f_{n}\left(z_{0}\right)=f\left(z_{0}\right)$ for a point $z_{0}$ of this domain, then $\lim _{n \rightarrow \infty} f_{n}(z)=f(z)$ for every point $z$ of this domain; see [P-Sz], Part 3, the solution to Problem 256, p. 362). Let $\varepsilon>0$ be such that $(-1 / 2,-1 / 2+\varepsilon]$ $\cap S=\emptyset$. Examining the behaviour of the functions $I_{m, \tau}(A)$ and $I_{\tau}(A)$ on $(-1 / 2,-1 / 2+\varepsilon]$, since the order of magnitude of $\Gamma(A, \tau, \tau)$ as $A \rightarrow-1 / 2+0$ is $1 /(A+1 / 2)$ for $\tau>0$, and $1 /(A+1 / 2)^{3}$ for $\tau=0$, taking into account Lemmas 6-9, (11) and the definition of $I_{\tau}^{+}(A)$ (see below (11)) we obtain the following lemma for the case $\tau \geq 0$.

Lemma 10. Let $\tau \geq 0$ or $0<i \tau<1 / 2$. Then the sum

$$
8 p_{N} \sqrt{\pi} s^{3 / 4} \sum_{\substack{P Q=T_{0} \\ P>0,(N, P)=1}} \frac{|Q|^{1 / 2}}{P}\left(\frac{D}{P}\right) \sum_{\substack{f_{j} \in V_{1 / 2}^{*}(4 N) \\ 2 t_{j}=\tau}} \bar{b}_{j, \infty}\left(\frac{s}{D}\right) b_{j, \infty}\left(D Q^{2}\right)
$$

equals

$$
\sum_{L|N, L| T_{0}} \frac{\left(\frac{D}{L}\right)}{L} \mu(L) \sum_{\substack{u \in U_{N / L} \\ t_{u}=\tau}}\left(\sum_{Q \in \Lambda_{N / L, s}} \omega_{D}(Q) \int_{C_{Q, N / L}} \bar{u} d S\right) a_{u}\left(-\frac{T_{0}}{L}\right) .
$$

Proof. We have proved this above for $\tau \geq 0$. The remaining case is similar; using e.g. the functions $I_{m, 1}(A)$ and $I_{1}(A)$, and examining their residues in the half-plane $\{\operatorname{Re} A>-1 / 2\}$ we get the result for the exceptional eigenvalues, i.e. for $0<i \tau<1 / 2$.

8. The proof of Theorem 1, Theorem 2 and its Corollary. Multiplying the equation of Lemma 10 by $W_{1 / 2+i \tau}\left(-T_{0} z\right)$ (where $z \in H$ ), and 
summing over $T_{0} \neq 0$ we get

$$
\begin{aligned}
8 p_{N} & \sqrt{\pi} s^{3 / 4} \sum_{\substack{f_{j} \in V_{1 / 2}^{*}(4 N) \\
2 t_{j}=\tau}} \bar{b}_{j, \infty}\left(\frac{s}{D}\right) \operatorname{Sh}_{D} f_{j}(z) \\
= & \sum_{L \mid N} \frac{\left(\frac{D}{L}\right)}{L} \mu(L) \sum_{\substack{u \in U_{N / L} \\
t_{u}=\tau}}\left(\sum_{Q \in \Lambda_{N / L, s}} \omega_{D}(Q) \int_{C_{Q, N / L}} \bar{u} d S\right) u(L z) .
\end{aligned}
$$

Here the summation is over any orthonormal basis $\left\{f_{j}\right\}$ of the $\Delta_{1 / 2}$-eigenspace of $V_{1 / 2}^{*}(4 N)$ with eigenvalue $-1 / 4-(\tau / 2)^{2}$; denote this subspace (finite-dimensional) by $W=W_{4 N, 1 / 2, \tau}$. Since $s / D \equiv 0,1(\bmod 4), D Q^{2} \equiv$ $0,1(\bmod 4)$ and $s / D>0, D Q^{2}>0$, we can factor by the subspace of functions whose $n$th Fourier coefficient is 0 for every $n>0$ with $n \equiv 0,1(\bmod 4)$. More precisely let $W=W_{0} \oplus W_{1}$, where

$$
W_{0}=\left\{f \in W: b_{f, \infty}(n)=0 \text { for } n>0, n \equiv 0,1(\bmod 4)\right\},
$$

and $W_{1}$ is the orthogonal complement of $W_{0}$ in $W$. Then we can take for the orthonormal basis $\left\{f_{j}\right\}$ of $W$ the union of an orthonormal basis of $W_{0}$ and of $W_{1}$. This shows (since $u(L z)$ is a cusp form for $\Gamma_{0}(N)$ if $u \in U_{N / L}$ ) that

$$
\sum_{f_{j} \in W_{1}} \bar{b}_{j, \infty}\left(\frac{s}{D}\right) \operatorname{Sh}_{D} f_{j}(z)
$$

is a cusp form for $\Gamma_{0}(N)$. Now, if $D$ is fixed, $s / D$ may be any positive integer congruent to 0 or $1 \bmod 4$, and the $\operatorname{dim} W_{1} \times \infty$ matrix $\left(\left\{f_{j}\right\}\right.$ is an orthonormal basis of $W$ )

$$
\left\{\bar{b}_{j, \infty}(n): f_{j} \in W_{1}, n>0, n \equiv 0,1(\bmod 4)\right\}
$$

has rank $\operatorname{dim} W_{1}$, because if $\sum_{j, f_{j} \in W_{1}} \alpha_{j} \bar{b}_{j, \infty}(n)=0$ with some complex numbers $\alpha_{j}$ for every $n>0, n \equiv 0,1(\bmod 4)$, then $f=\sum_{j, f_{j} \in W_{1}} \bar{\alpha}_{j} f_{j} \in$ $W_{0}$, so $f \in W_{0} \cap W_{1}$, i.e. $f=0$, and hence every coefficient $\alpha_{j}$ is $0\left(\left\{f_{j}\right\}\right.$ is an independent system). We conclude that for each $f_{j} \in W_{1}$ the function $\mathrm{Sh}_{D} f_{j}$ is a cusp form for $\Gamma_{0}(N)$. For $f_{j} \in W_{0}$ this is obvious, since then $\mathrm{Sh}_{D} f_{j}=0$. So Theorem 1 is proved.

Up to this point we have not used the Hecke operators, but it is obvious that we may assume in (14) that our cusp forms (either of weight $1 / 2$ or of weight 0$)$ are Hecke eigenforms. So assume that every $f_{j}$ is an eigenfunction of $T_{p^{2}}$ for every $p$ with $(p, 4 N)=1$, and every $u \in U_{N}$ is an eigenfunction of the Hecke operators $H_{p}$ for $(p, 4 N)=1$. This is possible, since the Hecke operators $T_{p^{2}}$ for $(p, 4 N)=1$ are self-adjoint, they commute with each other, with $\Delta_{1 / 2}$, and if $N$ is odd, they also commute with $L$, and the analogous statements are true for $H_{p}$ with $(p, 4 N)=1$. 
Now, let $w \in U_{N}$ be a newform for $\Gamma_{0}(N)\left(w \in U_{N}\right.$, so its square integral is 1 ), and choose $\tau=t_{w}$. Then, by definition, $w$ is orthogonal to any $u(L z)$, where $u \in U_{N / L}, L>1$; and of course $w$ is orthogonal to any $u \in U_{N}$ if $u \neq w$. On the other hand, it is well known (and very easily proved using the action on Fourier coefficients at $\infty$ ) that $\mathrm{Sh}_{D}$ commutes with the Hecke operators, i.e. $\operatorname{Sh}_{D} \circ T_{p^{2}}=H_{p} \circ \operatorname{Sh}_{D}$ for any $(p, 4 N)=1$, which implies that

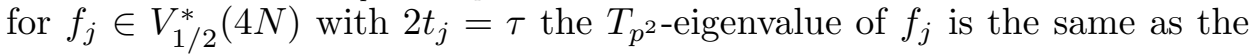
$H_{p}$-eigenvalue of $\operatorname{Sh}_{D} f_{j}$. So (since $H_{p}$ is self-adjoint for $(p, 4 N)=1$ ), $w$ is orthogonal to $\mathrm{Sh}_{D} f_{j}$ if there is a $(p, 4 N)=1$ for which $T_{p^{2}} f_{j} \neq \lambda_{w}(p) f_{j}$, with the notation $H_{p} w=\lambda_{w}(p) w$. The strong multiplicity one theorem for $\Gamma_{0}(N)$ shows that if $T_{p^{2}} f_{j}=\lambda_{w}(p) f_{j}$, i.e. $H_{p}\left(\operatorname{Sh}_{D} f_{j}\right)=\lambda_{w}(p) \operatorname{Sh}_{D} f_{j}$ for $(p, 4 N)=1$, then $\mathrm{Sh}_{D} f_{j}$ is a constant multiple of $w$. Since the first Fourier coefficient of $\operatorname{Sh}_{D} f_{j}$ is $b_{j, \infty}(D)$, this means $\operatorname{Sh}_{D} f_{j}(z)=b_{j, \infty}(D) w(z) / a_{w}(1)$. These considerations together with (14) imply

$$
\begin{aligned}
& \left(\sum_{Q \in \Lambda_{N, s}} \omega_{D}(Q) \int_{C_{Q, N}} \bar{w} d S\right) w(z) \\
& =8 p_{N} \sqrt{\pi} s^{3 / 4} \sum_{\substack{f_{j} \in V_{1 / 2}^{*}(4 N) \\
\operatorname{Sh}_{D} f_{j}(z)=b_{j, \infty}(D) w(z) / a_{w}(1)}} \bar{b}_{j, \infty}\left(\frac{s}{D}\right) b_{j, \infty}(D) \frac{w(z)}{a_{w}(1)} .
\end{aligned}
$$

Then (15) (dividing by $w$ and then conjugating) implies Theorem 2, upon writing $\varphi$ in place of $w / a_{w}(1)$.

Proof of the Corollary to Theorem 2. For $l^{\alpha} \| N(l$ is a prime, $\alpha \geq 1)$ we introduce the Atkin-Lehmer involution $W_{l^{\alpha}}($ see $[\mathrm{A}-\mathrm{L}])$ by

$$
W_{l^{\alpha}}=\frac{1}{\sqrt{l^{\alpha}}}\left(\begin{array}{cc}
l^{\alpha} & a \\
N & l^{\alpha} b
\end{array}\right),
$$

where $a, b$ are integers with $l^{2 \alpha} b-N a=l^{\alpha}$. It is well known that $W_{l^{\alpha}} \Gamma_{0}(N) W_{l^{\alpha}}$ $=\Gamma_{0}(N), W_{l^{\alpha}}^{2} \in \Gamma_{0}(N)$, and $W_{l^{\alpha}}$ commutes with the Hecke operators $T_{p}$ for $(p, N)=1$. Hence, if $\varphi$ is a newform for $\Gamma_{0}(N)$, then

$$
\varphi\left(W_{l^{\alpha}} z\right)=w_{l^{\alpha}} \varphi(z)
$$

with $w_{l^{\alpha}}= \pm 1$.

We will take $s=D^{2}$ in Theorem 2. A complete set of representatives of the $\Gamma_{0}(N)$-equivalence classes of $Q_{N, D^{2}}$ is

$$
\left\{Q_{\mu}(\tilde{X}, \tilde{Y}):\left(\begin{array}{c}
\tilde{X} \\
\widetilde{Y}
\end{array}\right)=W_{t}\left(\begin{array}{c}
X \\
Y
\end{array}\right), \mu(\bmod D), t \mid N,(t, N / t)=1\right\},
$$

where $Q_{\mu}(X, Y)=D X Y+\mu Y^{2}$, and if $t=\prod_{i=1}^{r} l_{i}^{\alpha_{i}}$ is the prime factorization of $t$, then $W_{t}=\prod_{i=1}^{r} W_{l_{i}^{\alpha_{i}}}$ (the order in the product is not important, 
because if $l_{1} \neq l_{2}$ then

$$
W_{l_{1}^{\alpha_{1}}} W_{l_{2}^{\alpha_{2}}} \Gamma_{0}(N)=\frac{1}{\sqrt{l_{1}^{\alpha_{1}} l_{2}^{\alpha_{2}}}}\left(\begin{array}{cc}
l_{1}^{\alpha_{1}} l_{2}^{\alpha_{2}} & a \\
N & l_{1}^{\alpha_{1}} l_{2}^{\alpha_{2}} b
\end{array}\right) \Gamma_{0}(N),
$$

where $a, b$ are integers with $\left.l_{1}^{2 \alpha_{1}} l_{2}^{2 \alpha_{2}} b-N a=l_{1}^{\alpha_{1}} l_{2}^{\alpha_{2}}\right)$. This is stated in [Ko2] for $N$ squarefree, but it is easily seen to be true in general. (One can prove it in the following way. Firstly, these forms are inequivalent, which follows from the properties of the Atkin-Lehmer involutions, and from the fact that if $Q_{\mu_{1}}(\tilde{X}, \tilde{Y})=Q_{\mu_{2}}(X, Y)$ with $\left(\begin{array}{c}\tilde{X} \\ \tilde{Y}\end{array}\right)=\gamma W_{t}\left(\begin{array}{c}X \\ Y\end{array}\right)$ and $\gamma \in \Gamma_{0}(N)$, then $t=1$ and $\mu_{1} \equiv \mu_{2}(\bmod D)$ (because we must have $\left.\gamma W_{t} \infty=\infty\right)$. So it is enough to prove that the number of equivalence classes is $D 2^{\nu(N)}$, where $\nu(N)$ is the number of distinct prime factors of $N$. But this is true, because for $N=1$ the statement is easy, and for general $N$ the $\Gamma_{0}(N)$-equivalence classes of $Q_{1, D^{2}}$ are

$$
\left\{Q_{\mu}(\tilde{X}, \tilde{Y}):\left(\begin{array}{c}
\tilde{X} \\
\widetilde{Y}
\end{array}\right)=\gamma_{i}\left(\begin{array}{l}
X \\
Y
\end{array}\right)\right\}
$$

where we can take $\mu=0, N, 2 N, \ldots,(D-1) N$, and $\left\{\gamma_{i}\right\}$ is a set of representatives of left cosets $\Gamma_{0}(N)$ in $\Gamma_{0}(1)$. We can take for $\left\{\gamma_{i}\right\}$ a set

$$
\left\{\left(\begin{array}{ll}
u & \cdot \\
v & \cdot
\end{array}\right) \in \Gamma_{0}(1): u \mid N, v\left(\bmod \frac{N}{u}\right)\right\}
$$

and it is easy to see that we get a class in $Q_{N, D^{2}}$ exactly when $(u, N / u)=1$ and $N / u \mid v$.)

It is obvious that if $Q \in Q_{N, D^{2}}, t \mid N,(t, N / t)=1$ and $\widetilde{Q}(X, Y)=$ $Q(\widetilde{X}, \widetilde{Y})$ with $\left(\begin{array}{c}\widetilde{X} \\ \widetilde{Y}\end{array}\right)=W_{t}\left(\begin{array}{c}X \\ Y\end{array}\right)$, then $\widetilde{Q} \in Q_{N, D^{2}}$ and $\omega_{D}(\widetilde{Q})=\left(\frac{D}{t}\right) \omega_{D}(Q)$. On the other hand, for $Q \in Q_{N, D^{2}}$ the curve $C_{Q, N}$ is the whole semicircle. So (taking $w_{t}=\prod_{i=1}^{r} w_{l_{i}^{\alpha_{i}}}$ for $t=\prod_{i=1}^{r} l_{i}^{\alpha_{i}}$ ) we get

$$
\begin{aligned}
\sum_{Q \in \Lambda_{N, s}} \omega_{D}(Q) & \int_{C_{Q, N}} \varphi d S \\
& =\sum_{\mu(\bmod D)} \sum_{\substack{t \mid N \\
(t, N / t)=1}}\left(\frac{D}{\mu}\right)\left(\frac{D}{t}\right) w_{t} \int_{-\mu / D}^{-\mu / D+i \infty} \varphi(z) \frac{d y}{y} .
\end{aligned}
$$

If Re $s$ is large, then

$$
\begin{aligned}
& \sum_{\mu(\bmod D)}\left(\frac{D}{\mu}\right) \int_{-\mu / D}^{-\mu / D+i \infty} \varphi(z) y^{s} \frac{d y}{y} \\
& =2 \pi^{-s-1 / 2} D^{1 / 2} \Gamma\left(\frac{s+1 / 2+i t}{2}\right) \Gamma\left(\frac{s+1 / 2-i t}{2}\right) L(\varphi, D ; s+1 / 2),
\end{aligned}
$$


by the Fourier expansion of $\varphi,\left(\frac{D}{\mu}\right)=\left(\frac{D}{-\mu}\right)($ as $D>0)$,

$$
\sum_{\mu(\bmod D)}\left(\frac{D}{\mu}\right) e\left(\frac{\mu k}{D}\right)=\left(\frac{D}{k}\right) D^{1 / 2}
$$

([La], Satz 215), and

$$
\int_{0}^{\infty} K_{\nu}(x) x^{S-1} d x=2^{S-2} \Gamma\left(\frac{S+\nu}{2}\right) \Gamma\left(\frac{S-\nu}{2}\right) .
$$

By analytic continuation, if $N=\prod_{i=1}^{R} l_{i}^{\alpha_{i}}$ we have

$$
\begin{aligned}
\sum_{Q \in \Lambda_{N, s}} \omega_{D}(Q) \int_{C_{Q, N}} \varphi d S= & 2 \pi^{-1 / 2} D^{1 / 2}\left(\prod_{i=1}^{R}\left(1+\left(\frac{D}{l_{i}^{\alpha_{i}}}\right) w_{l_{i}^{\alpha_{i}}}\right)\right) \\
& \times \Gamma\left(\frac{1 / 2+i t}{2}\right) \Gamma\left(\frac{1 / 2-i t}{2}\right) L(\varphi, D ; 1 / 2) .
\end{aligned}
$$

Together with Theorem 2 (with $s=D^{2}$ ) this implies the Corollary.

\section{Lemmas on special functions}

Lemma 11. Let $\operatorname{Re} M>1 / 2$. Then

$$
\lim _{y \rightarrow 0+0} \int_{-\infty}^{\infty} \frac{J_{M}\left(\sqrt{y^{2}+R^{2}}\right)}{\left(\sqrt{y^{2}+R^{2}}\right)^{3 / 2}} e^{-i R} d R=2\left(\frac{2}{\pi}\right)^{1 / 2} \frac{1}{M^{2}-1 / 4} \cos \frac{\pi}{2}\left(M-\frac{1}{2}\right) .
$$

Proof. By [W], p. 385, (2), we have

$$
\begin{aligned}
& \int_{0}^{\infty} e^{-a R} J_{\nu}(b R) R^{\mu-1} d R \\
& =\frac{(b / 2)^{\nu} \Gamma(\mu+\nu)}{a^{\mu+\nu} \Gamma(\nu+1)} F\left(\frac{\mu+\nu}{2}, \frac{\mu+\nu+1}{2} ; \nu+1 ;-\frac{b^{2}}{a^{2}}\right)
\end{aligned}
$$

if $\operatorname{Re}(\mu+\nu)>0, \operatorname{Re} a>0, \operatorname{Re}(a+i b)>0, \operatorname{Re}(a-i b)>0$. Now substitute $b=1, \mu=-1 / 2, \nu=M$, and let $a \rightarrow t i(t<-1$ or $t>1)$ inside the right half-plane. By taking the limit the formula is still valid for $a=t i$. We let $t \rightarrow 1+0(t \rightarrow-1-0)$, and use

$$
\lim _{z \rightarrow 1-0} F(\alpha, \beta, \gamma ; z)=\frac{\Gamma(\gamma) \Gamma(\gamma-\alpha-\beta)}{\Gamma(\gamma-\alpha) \Gamma(\gamma-\beta)}
$$

for $\operatorname{Re}(\gamma-\alpha-\beta)>0([\mathrm{Le}]$, p. 294$)$, and $\Gamma(s) \Gamma(s+1 / 2)=\pi^{1 / 2} 2^{1-2 s} \Gamma(2 s)$, getting the assertion. 
Lemma 12. For $y>0$ let

$$
\varphi_{M}(y)=\int_{-\infty}^{\infty} \frac{J_{M}\left(\sqrt{y^{2}+R^{2}}\right)}{\left(\sqrt{y^{2}+R^{2}}\right)^{3 / 2}} e^{-i R} d R
$$

( $M$ is any complex number). Then

$$
y^{2} \varphi_{M}^{(2)}(y)+2 y \varphi_{M}^{(1)}(y)-\varphi_{M}(y)\left(y^{2}+M^{2}-1 / 4\right)=-2(2 / \pi)^{1 / 2} \cos \frac{\pi}{2}(M-1 / 2) .
$$

Proof. Put $R_{M}(x)=J_{M}(x) / x^{3 / 2}$ for $x>0$. Then

$$
R_{M}^{(2)}(x)+4 \frac{R_{M}^{(1)}(x)}{x}+R_{M}(x)=\left(M^{2}-9 / 4\right) \frac{R_{M}(x)}{x^{2}} .
$$

We will repeatedly use the following claim:

CLAIM. If $j(R)$ is a smooth function on the positive real axis with $j^{(k)}(R)$ $=O_{k}\left(1 / R^{2}\right)$ as $R \rightarrow \infty(k=0,1,2, \ldots)$, and

$$
F(y)=\int_{-\infty}^{\infty} j\left(\sqrt{y^{2}+R^{2}}\right) e^{-i R} d R \quad \text { for } y>0
$$

then

$$
\begin{gathered}
\frac{F^{(1)}(y)}{y}=\int_{-\infty}^{\infty} \frac{j^{(1)}\left(\sqrt{y^{2}+R^{2}}\right)}{\sqrt{y^{2}+R^{2}}} e^{-i R} d R \\
F^{(2)}(y)-F(y)-\frac{F^{(1)}(y)}{y}=\int_{-\infty}^{\infty} j^{(2)}\left(\sqrt{y^{2}+R^{2}}\right) e^{-i R} d R
\end{gathered}
$$

Proof. Assertion (i) follows by differentiating the definition of $F(y)$ under the integral sign; (ii) follows by a further differentiation, using (i), and applying partial integration two times for the definition of $F(y)$.

By repeated application of this claim, and using

$$
\frac{\left(R_{M}^{(1)}(x) / x\right)^{(1)}}{x}=\frac{R_{M}^{(2)}(x)}{x^{2}}-\frac{R_{M}^{(1)}(x)}{x^{3}}
$$

we have

$$
\frac{\left(\varphi_{M}^{(1)}(y) / y\right)^{(1)}}{y}=\int_{-\infty}^{\infty} \frac{R_{M}^{(2)}\left(\sqrt{y^{2}+R^{2}}\right)}{\left(\sqrt{y^{2}+R^{2}}\right)^{2}}-\frac{R_{M}^{(1)}\left(\sqrt{y^{2}+R^{2}}\right)}{\left(\sqrt{y^{2}+R^{2}}\right)^{3}} e^{-i R} d R .
$$

Similarly, with the notation

$$
\gamma_{M}(y)=\varphi_{M}^{(2)}(y)+3 \frac{\varphi_{M}^{(1)}(y)}{y}
$$


using also the differential equation for $R_{M}(x)$, we obtain

$$
\gamma_{M}(y)=\left(M^{2}-9 / 4\right) \int_{-\infty}^{\infty} \frac{R_{M}\left(\sqrt{y^{2}+R^{2}}\right)}{\left(\sqrt{y^{2}+R^{2}}\right)^{2}} e^{-i R} d R .
$$

Since

$$
\left(\frac{R_{M}(x)}{x^{2}}\right)^{(2)}+3 \frac{\left(R_{M}(x) / x^{2}\right)^{(1)}}{x}=\frac{R_{M}^{(2)}(x)}{x^{2}}-\frac{R_{M}^{(1)}(x)}{x^{3}},
$$

this implies in view of (17) that

$$
\frac{1}{M^{2}-9 / 4}\left(\gamma_{M}^{(2)}(y)+2 \frac{\gamma_{M}^{(1)}(y)}{y}-\gamma_{M}(y)\right)=\frac{\left(\varphi_{M}^{(1)}(y) / y\right)^{(1)}}{y},
$$

so we have obtained a fourth order differential equation for $\varphi_{M}(y)$. Multiplying by $y$ and using $y \gamma_{M}(y)=\left(y \varphi_{M}^{(1)}(y)+2 \varphi_{M}(y)\right)^{(1)}$ we get

$$
\left(M^{2}-9 / 4\right) \frac{\varphi_{M}^{(1)}(y)}{y}-y \gamma_{M}^{(1)}(y)-\gamma_{M}(y)+y \varphi_{M}^{(1)}(y)+2 \varphi_{M}(y)=c_{1, M}
$$

with some constant $c_{1, M}$, because the derivative of the left-hand side is 0 . From this, as above, we get

$$
\left(M^{2}-9 / 4\right) \varphi_{M}(y)-y^{2} \gamma_{M}(y)+y \varphi_{M}^{(1)}(y)+2 \varphi_{M}(y)+y^{2} \varphi_{M}(y)=\frac{c_{1, M}}{2} y^{2}+c_{2, M},
$$

because the derivative of the left-hand side is $c_{1, M} y$.

Now, the left-hand side is

$$
-\left(y^{2} \varphi_{M}^{(2)}(y)+2 y \varphi_{M}^{(1)}(y)-\varphi_{M}(y)\left(y^{2}+M^{2}-1 / 4\right)\right) .
$$

It is easy to see that $\varphi_{M}(y), \varphi_{M}^{(1)}(y), \varphi_{M}^{(2)}(y)=O(1 / y)$ as $y \rightarrow \infty$, so $c_{1, M}$ is 0 . On the other hand, if for example $M$ is a large enough positive number, then (by Lemma 11) the limit of this left-hand side as $y \rightarrow 0+0$ is $2(2 / \pi)^{1 / 2} \cos \frac{\pi}{2}(M-1 / 2)$, which proves the lemma.

Corollary. Let

$$
\begin{aligned}
\eta_{M}(y) & =\varphi_{M}(y) \cos \frac{\pi}{2}(M+1 / 2) \\
\psi_{M}(y) & =\frac{(\Gamma(1 / 4+M / 2) \Gamma(1 / 4-M / 2))^{-1}}{\sin \frac{\pi}{2} M \cos \pi M}\left(\eta_{M}(y)-\eta_{-M}(y)\right) .
\end{aligned}
$$

Then

$$
\psi_{M}(y)=-\frac{2}{\pi^{3 / 2}} y^{-1 / 2} K_{M}(y) \cos \frac{\pi}{2} M .
$$

Proof. It is clear by the lemma that

$$
y^{2} \psi_{M}^{(2)}(y)+2 y \psi_{M}^{(1)}(y)-\psi_{M}(y)\left(y^{2}+M^{2}-1 / 4\right)=0,
$$

so

$$
y^{2}\left(y^{1 / 2} \psi_{M}(y)\right)^{(2)}+y\left(y^{1 / 2} \psi_{M}(y)\right)^{(1)}-\left(y^{2}+M^{2}\right)\left(y^{1 / 2} \psi_{M}(y)\right)=0,
$$


which means that $y^{1 / 2} \psi_{M}(y)$ is a constant multiple of $K_{M}(y)$, because obviously $y^{1 / 2} \psi_{M}(y)=O(1)$ as $y \rightarrow \infty$. Now we determine the constant. Let, for example, $M \in(9,10)$, and $y \rightarrow 0+0$. Then, by the power series expansion of the $J$-function and the $K$-function, and since

$$
\int_{-\infty}^{\infty}\left(1+R^{2}\right)^{-\frac{1}{2}(M+3 / 2)} d R=\pi^{1 / 2} \frac{\Gamma(M / 2+1 / 4)}{\Gamma(M / 2+3 / 4)}
$$

(see (3.18) of [I1]), as $y \rightarrow 0+0$ we have

$$
\begin{aligned}
y^{1 / 2} \psi_{M}(y) \sim & -\cos \frac{\pi}{2}\left(-M+\frac{1}{2}\right) \frac{(\Gamma(1 / 4+M / 2) \Gamma(1 / 4-M / 2))^{-1}}{\sin \frac{\pi}{2} M \cos \pi M} \\
& \times \frac{2^{M} y^{-M}}{\Gamma(-M+1)} \pi^{1 / 2} \frac{\Gamma(M / 2+1 / 4)}{\Gamma(M / 2+3 / 4)}
\end{aligned}
$$

and

$$
K_{M}(y) \sim \frac{\pi}{2}(\sin \pi M)^{-1} \frac{2^{M} y^{-M}}{\Gamma(-M+1)} .
$$

Hence using $\sin 2 s=2 \sin s \cos s$ and $\Gamma(s) \Gamma(1-s)=\pi(\sin \pi s)^{-1}$ we get the result (by analytic continuation).

Lemma 13. If $M=3 / 2+2 t(t=0,1,2, \ldots)$, then $\varphi_{M}(y, \vartheta)+\varphi_{M}(y,-\vartheta)$ $=0$ for every $y \in(0, \infty), \vartheta \in(-\pi / 2, \pi / 2)$. (See the definition of $\varphi_{M}(y, \vartheta)$ in (5).)

Proof. In these cases $L_{M}(z)$ is an odd entire function, so

$$
z \mapsto\left(L_{M}\left(z+y \frac{\sin \vartheta}{\cos \vartheta}\right)+L_{M}\left(z-y \frac{\sin \vartheta}{\cos \vartheta}\right)\right) / z
$$

is an even entire function, hence the integrand is an entire function of $R$. We can change the line of integration from $(-\infty, \infty)$ to $(-i T-\infty,-i T+\infty)$, and it is easy to see that the integral tends to 0 as $T \rightarrow \infty$, because $J_{M}(z)=O\left(|z|^{-1 / 2} e^{|\operatorname{Im} z|}\right.$ ) as $|z| \rightarrow \infty$ (e.g. from the expression for $J_{M}(z)$ as an elementary function in case $M$ is half an odd integer; see [W], p. 53).

It is very likely that one could compute the integral of the following lemma explicitly, but an upper bound will suffice for our purposes.

LEMMA 14. Let $\mu$ be a given purely imaginary number, and let $B$ be a fixed positive constant. Then, if $A$ is a large enough positive number, and

$$
I_{\mu, A}(M, \vartheta)=\cos \frac{\pi}{2}(M+1 / 2) \int_{0}^{\infty} K_{\mu}(y) y^{A} \varphi_{M}(y, \vartheta) d y
$$

where 
$\varphi_{M}(y, \vartheta)$

$$
=\int_{0}^{\infty} L_{M}\left(\sqrt{\frac{y^{2}}{\cos ^{2} \vartheta}+R^{2}}+y \frac{\sin \vartheta}{\cos \vartheta}\right)\left(\frac{y^{2}}{\cos ^{2} \vartheta}+R^{2}\right)^{-1 / 2}\left(e^{i R}+e^{-i R}\right) d R
$$

and $L_{M}(x)=J_{M}(x) / \sqrt{x}$, then

$$
\begin{array}{r}
I_{\mu, A}(M, \vartheta)+I_{\mu, A}(M,-\vartheta)-\left(I_{\mu, A}(-M, \vartheta)+I_{\mu, A}(-M,-\vartheta)\right) \\
=O_{A, B, \mu}\left(e^{\pi|\operatorname{Im} M|}(1+|\operatorname{Im} M|)^{-B}\right)
\end{array}
$$

if $|\operatorname{Re} M| \leq A / 100$, uniformly in $\vartheta$ if $|\vartheta|$ is small enough (smaller than a positive constant depending on $A, B$ and $\mu$ ).

Proof. We will prove the desired upper bound for $I_{\mu, A}(M, \vartheta)-$ $I_{\mu, A}(-M,-\vartheta)$. Let $\operatorname{Im} M=t$; we assume $t \geq 2$.

We use the weak estimate

$$
J_{M}(z)=O\left(e^{(\pi / 2-\alpha) t} e^{|\operatorname{Im} z|} t^{A / 10}(1+1 /|z|)^{A / 10}\right)
$$

for $-\pi / 2<\alpha=\arg z<\pi / 2$ and $|\operatorname{Re} M| \leq A / 100$. By Phragmén-Lindelöf it is enough to prove this when $\operatorname{Re} M$ is an even integer, and in this case it follows easily from

$$
J_{\nu}(z)=\frac{(z / 2)^{\nu}}{\Gamma(\nu+1 / 2) \Gamma(1 / 2)} \int_{-1}^{1}\left(1-t^{2}\right)^{\nu-1 / 2} \cos z t d t \quad(\operatorname{Re} \nu>-1 / 2),
$$

and from the relation $J_{\nu-1}(z)+J_{\nu+1}(z)=2 \nu z^{-1} J_{\nu}(z)$.

Consider $I_{\mu, A}(M, \vartheta)$. Replace the path of integration $[0, \infty)$ in $y$ by $P_{1}=$ $\left[0,(c \log t) e^{i \alpha}\right]$ and $P_{2}=\left[(c \log t) e^{i \alpha}+0,(c \log t) e^{i \alpha}+\infty\right)$, where $c$ is a large and $\alpha$ a small positive constant so that $c \sin \alpha$ is also small. If $y \in P_{2}$, then we may integrate in $R$ e.g. on the same path as in $y$, and since $K_{\mu}(y)=$ $O\left(e^{-\operatorname{Re} y}\right)$, and $c$ is a large constant and $\vartheta$ is small, it is enough to deal with $P_{1}$.

If $y \in P_{1}$ is fixed, integrate in $R$ on the paths

$$
\begin{aligned}
Q_{1} & =\left[0,(c \log t) e^{i \alpha}-y \frac{\sin \vartheta}{\cos \vartheta}\right], \\
Q_{2} & =\left[(c \log t) e^{i \alpha}-y \frac{\sin \vartheta}{\cos \vartheta}+0,(c \log t) e^{i \alpha}-y \frac{\sin \vartheta}{\cos \vartheta}+\infty\right)
\end{aligned}
$$

(we fix $c$ and $\alpha$ first, and let $\vartheta$ be very small with respect to them). It is enough to deal with

$$
Q_{3}=\left[(c \log t) e^{i \alpha}-y \frac{\sin \vartheta}{\cos \vartheta}+t^{1-\varepsilon},(c \log t) e^{i \alpha}-y \frac{\sin \vartheta}{\cos \vartheta}+\infty\right)
$$

with a small $\varepsilon>0$, otherwise we are done by (18). But here the $e^{i R}$-part of the integral is small (push the line of integration upwards), and $\sqrt{y^{2} / \cos ^{2} \vartheta+R^{2}}$ is very close to $R$, so using Taylor's formula, substituting 
$R+y \sin \vartheta / \cos \vartheta \rightarrow R$, and handling $I_{\mu, A}(-M,-\vartheta)$ similarly, but "downwards", we can replace $I_{\mu, A}(M, \vartheta)-I_{\mu, A}(-M,-\vartheta)$ with a small error by an expression of the type (since we can now $y$-integrate over the real line)

$$
\begin{aligned}
\sum_{j=0}^{J} \sum_{t=0}^{T} \sum_{q=0}^{K} \frac{a_{j, t} b_{j, t, q}}{j !} & \sin ^{q} \vartheta \cos ^{-2 j-2 t-q} \vartheta \\
& \times\left(\int_{0}^{\infty} K_{\mu}(y) y^{A+2 j+2 t+q} e^{i y \sin \vartheta / \cos \vartheta} d y\right) I_{j, 2 t+q}(M),
\end{aligned}
$$

where $a_{j, t}, b_{j, t, q}$ are defined by the power series expansions

$$
\left(\frac{(1+x)^{1 / 2}-1}{x}\right)^{j}(1+x)^{-1 / 2}=\sum_{t=0}^{\infty} a_{j, t} x^{t}, \quad\left(\frac{1}{1-x}\right)^{1+j+2 t}=\sum_{q=0}^{\infty} b_{j, t, q} x^{q},
$$

and

$$
\begin{aligned}
I_{j, z}(M)= & \cos \frac{\pi}{2}\left(M+\frac{1}{2}\right) \int_{Q^{+}} \frac{L_{M}^{(j)}(R)}{R^{1+j+z}} e^{-i R} d R \\
& -(-1)^{z} \cos \frac{\pi}{2}\left(-M+\frac{1}{2}\right) \int_{Q^{-}} \frac{L_{-M}^{(j)}(R)}{R^{1+j+z}} e^{i R} d R
\end{aligned}
$$

with the notations

$$
\begin{aligned}
& Q^{+}=\left[(c \log t) e^{i \alpha}+t^{1-\varepsilon},(c \log t) e^{i \alpha}+\infty\right), \\
& Q^{-}=\left[(c \log t) e^{-i \alpha}+t^{1-\varepsilon},(c \log t) e^{-i \alpha}+\infty\right) .
\end{aligned}
$$

So it suffices to prove that $I_{j, z}(M)$ is small for all $j, z \geq 0$. We choose a large constant $E$ and a large positive integer $N$. Then the multiplier $\left(1-e^{-E R}\right)^{N}$ is practically 1 on $Q^{ \pm}$; with this factor we can transform back the integration to $[0, \infty)$, and it is enough to show that (remember $\operatorname{Im} M \geq 2$ )

$$
\begin{aligned}
\int_{0}^{\infty} \frac{J_{M}^{(j)}(R) e^{-i R}-i(-1)^{z} J_{-M}^{(j)}(R) e^{i R}}{R^{3 / 2+j+z}}\left(1-e^{-E R}\right)^{N} d R & \\
& =O\left(e^{(\pi / 2) \operatorname{Im} M}(\operatorname{Im} M)^{-C}\right)
\end{aligned}
$$

for every $C>0$. For $j=0$ this follows easily from (16), and in view of $J_{\nu-1}(R)-J_{\nu+1}(R)=2 J_{\nu}^{(1)}(R)$ this proves the lemma.

LeMma 15. Let $\varphi$ be a smooth function on $(0, \infty)$ such that $\varphi(x)=$ $O\left(x^{A}\right)$ with some large constant $A$ as $x \rightarrow 0$, and $\varphi(x)=O\left(x^{-1 / 2}\right)$ as $x \rightarrow \infty$. Assume that $\widetilde{\varphi}(1 / 2+2 j)=0$ for $j=1,2, \ldots$, and $\varphi^{*}(t)-\varphi^{*}(-t)=$ $O\left(e^{2 \pi|t|}(1+|t|)^{-B}\right)$ in a strip $|\operatorname{Im} t|<C$ with some large constants $B$ and $C$. 
If

$$
\int_{-\infty}^{\infty} \widehat{\varphi}(t) \Gamma(1 / 4+i t) \Gamma(1 / 4-i t)(\operatorname{sh} \pi t) t d t=0
$$

then $\varphi$ satisfies the conditions of Theorem $\mathrm{B}$, i.e. for some $\varepsilon>0, \varphi(x)=$ $O\left(x^{-1-\varepsilon}\right)$ and $\varphi^{(l)}(x)=O\left(x^{-2-\varepsilon}\right)$ for $l=1,2,3$ as $x \rightarrow \infty$.

Proof. For $x>0$ define

$$
\psi(x)=-\frac{2}{\pi^{2} e(3 / 8)} \int_{-\infty}^{\infty} \frac{J_{2 i t}(x) \cos (\pi i t+\pi / 4) \widehat{\varphi}(t) \Gamma(1 / 4+i t) \Gamma(1 / 4-i t)}{\operatorname{ch} \pi t} t d t .
$$

Then it is easy to check that $\psi(0)=\psi^{(1)}(0)=0$ (moving the line of integration to $\operatorname{Im} t=-(1 / 2+\varepsilon)$ and using $\widehat{\varphi}(-i / 2)=0)$, and $\psi(x)=O\left(x^{-1-\varepsilon}\right)$ and $\varphi^{(l)}(x)=O\left(x^{-2-\varepsilon}\right.$ ) for $l=1,2,3$ as $x \rightarrow \infty$ (using, for $x$ very large with respect to $t$, the asymptotic formula for the $J$-function and its derivatives, and taking into account (19), we get in fact much stronger estimates). So it is enough to prove that $\varphi=\psi$. But the Corollary to Lemma A.9 below shows that $\widehat{\varphi}=\widehat{\psi}$ and $\widehat{\psi}(1 / 2+2 j)=0$ for $j=1,2, \ldots$ So for the function $d(x)=(\varphi-\psi)(x)$ we have

$$
\int_{-\infty}^{\infty} d(x) F(x, 2 t,-1 / 2) \frac{d x}{x}=0
$$

for each real $t$ with

$$
F(x, 2 t,-1 / 2)=J_{2 i t}(x) \cos \pi(i t+1 / 4)-J_{-2 i t}(x) \cos \pi(-i t+1 / 4),
$$

and

$$
\int_{-\infty}^{\infty} d(x) J_{2 j-1 / 2}(x) \frac{d x}{x}=0 \quad \text { with } j=1,2, \ldots
$$

But then $d \equiv 0$, because $d$ is easily seen to be orthogonal in $L^{2}\left((0, \infty), x^{-1} d x\right)$ to any compactly supported smooth function by the Appendix in $[\mathrm{K}]$.

COROLlary. There is a fixed function $\varphi_{0}$ satisfying the conditions of Lemma 15 apart from (19) (writing $\varphi_{0}$ in place of $\varphi$ there), such that if $\varphi$ is any other such function then Theorem $\mathrm{B}$ is applicable to the function

$$
\varphi(x)-\left(\int_{-\infty}^{\infty} \widehat{\varphi}(t) \Gamma(1 / 4+i t) \Gamma(1 / 4-i t)(\operatorname{sh} \pi t) t d t\right) \varphi_{0}(x) .
$$

Proof. We take $\varphi_{0}$ with

$$
\int_{-\infty}^{\infty} \widehat{\varphi}_{0}(t) \Gamma(1 / 4+i t) \Gamma(1 / 4-i t)(\operatorname{sh} \pi t) t d t=1
$$


(if this integral is 0 for every function satisfying the desired conditions, then we can take $\left.\varphi_{0} \equiv 0\right)$.

\section{APPENDIX: KUZNETSOV'S FORMULA FOR KOHNEN'S SUBSPACE}

Here we prove Theorem B stated in Section 3. The proof is based on the ordinary Kuznetsov formula for Maass forms of weight $1 / 2$ (proved by Proskurin $[\mathrm{P}]$ ) and the fact that (just as in the holomorphic case) Kohnen's subspace is the invariant space of a certain self-adjoint operator $L$ satisfying a quadratic equation.

In Section A.1 we give some notations, in Section A.2 we state the basic properties of the operator $L$ and determine its action on Fourier coefficients (which is important for the proof), and in Section A.3 we compute the relevant Kloosterman sums. After these preliminaries we can easily prove Theorem B in Section A.4. We have there a general test function on the Kloosterman sum side and not on the spectral side; Section A.5 deals with the inversion of the integral transformation involved.

A.1. Further notations. We define the symbol $\left(\frac{c}{d}\right)$ for $c$ an integer, and $d$ an odd integer. For $d>0$ this is the usual Jacobi symbol, and we extend it by the formulas

$$
\begin{gathered}
\left(\frac{c}{d}\right)=\frac{c}{|c|}\left(\frac{c}{-d}\right) \quad \text { for } c \neq 0 \\
\left(\frac{0}{d}\right)=1 \text { for } d= \pm 1, \quad\left(\frac{0}{d}\right)=0 \text { otherwise. }
\end{gathered}
$$

A few basic properties are the following. We have $\left(\frac{c}{d}\right)=0$ for $(c, d)>1$, and the symbol is completely multiplicative in both variables. If $n$ is odd, then $\left(\frac{-1}{n}\right)=(-1)^{(n-1) / 2}$ and $\left(\frac{2}{n}\right)=(-1)^{\left(n^{2}-1\right) / 8}$. If $A$ is an integer, then for any integer $c$ and $d$ odd one has $\left(\frac{c}{d}\right)=\left(\frac{c}{d+A(4 c)}\right)$, but if $4 \mid c$, then $\left(\frac{c}{d}\right)=\left(\frac{c}{d+A c}\right)$ is also true. If $A$ and $c$ are any integers, $d$ is odd and $d>0$, then $\left(\frac{c}{d}\right)=\left(\frac{c+A d}{d}\right)$. Finally, the extended quadratic reciprocity law states that if $p$ and $q$ are odd integers, $(p, q)=1$, and $p>0$, then

$$
\left(\frac{p}{q}\right)\left(\frac{q}{p}\right)=(-1)^{(p-1) / 2 \cdot(q-1) / 2} \text {. }
$$

(Proof. For $q>0$ this is well known, so we assume $q<0$. Then

$$
\left(\frac{p}{q}\right)=\left(\frac{p}{-q}\right), \quad\left(\frac{q}{p}\right)=(-1)^{(p-1) / 2}\left(\frac{-q}{p}\right),
$$

hence $($ as $-q>0)$ 


$$
\left(\frac{p}{q}\right)\left(\frac{q}{p}\right)=(-1)^{(p-1) / 2}(-1)^{(p-1) / 2 \cdot(-q-1) / 2}=(-1)^{(p-1) / 2 \cdot(1-q) / 2},
$$

and $(-1)^{n}=(-1)^{-n}$ for every integer $n$.)

For a complex number $z \neq 0$ we set its argument in $(-\pi, \pi]$, and $\log z=$ $\log |z|+i \arg z$, where $\log |z|$ is real. We define the power $z^{s}$ for any $s \in \mathbb{C}$ by $z^{s}=\exp (s \log z)$.

For $g_{1}, g_{2} \in \mathrm{GL}^{+}(2, \mathbb{R})$, we define

$$
w\left(g_{1}, g_{2}\right)=j_{g_{1}}\left(g_{2} z\right)^{1 / 2} j_{g_{2}}(z)^{1 / 2} j_{g_{1} g_{2}}(z)^{-1 / 2} .
$$

The right-hand side is indeed independent of $z \in H$. Clearly $w= \pm 1$. One has

$$
f\left|g_{1} g_{2}=w\left(g_{1}, g_{2}\right)\left(f \mid g_{1}\right)\right| g_{2} .
$$

We will frequently (and sometimes tacitly) use the following lemma.

Lemma A.1. Let $g_{1}, g_{2} \in \mathrm{GL}^{+}(2, \mathbb{R}), g_{1}=\left(\begin{array}{ll}a_{1} & b_{1} \\ c_{1} & d_{1}\end{array}\right), g_{2}=\left(\begin{array}{ll}a_{2} & b_{2} \\ c_{2} & d_{2}\end{array}\right)$ and $g_{1} g_{2}=\left(\begin{array}{ll}A & B \\ C & D\end{array}\right)$.

(i) If $c_{2}>0$ and $C>0$, then $w\left(g_{1}, g_{2}\right)=1$.

(ii) If $c_{1}=0$ and $d_{1}>0$, or if $c_{2}=0$ and $d_{2}>0$ (i.e. $j_{g_{1}}(z)$ or $j_{g_{2}}(z)$ is a positive constant), then $w\left(g_{1}, g_{2}\right)=1$.

Proof. We use the identity $j_{g_{1} g_{2}}(z)=j_{g_{1}}\left(g_{2} z\right) j_{g_{2}}(z)$. Statement (ii) is trivial from this, but statement (i) also follows from $z=x+i, x \rightarrow \infty$ (because then $\arg j_{g_{1} g_{2}}(z) \rightarrow 0$ and $\arg j_{g_{2}}(z) \rightarrow 0$ ).

The function $\nu$ defined in Section 2 is a multiplier system on $\Gamma_{0}(4)$ (and on every $\left.\Gamma_{0}(4 N)\right)$ of weight $1 / 2$, in the sense that $|\nu|=1, \nu\left(\left(\begin{array}{cc}-1 & 0 \\ 0 & -1\end{array}\right)\right)=$ $e^{-\pi i / 2}$, and

$$
\nu\left(g_{1} g_{2}\right)=w\left(g_{1}, g_{2}\right) \nu\left(g_{1}\right) \nu\left(g_{2}\right)
$$

for every $g_{1}, g_{2} \in \Gamma_{0}(4)$.

Let $N$ be a positive integer. For a cusp $a$ of $\Gamma_{0}(4 N)$ denote the stability group of $a$ by $\Gamma_{a}=\left\{\gamma \in \widehat{\Gamma}_{0}(4 N): \gamma a=a\right\}$. The scaling matrix of $a$ is an element $\sigma_{a} \in \mathrm{SL}(2, \mathbb{R})$ such that $\sigma_{a} \infty=a$ and $\sigma_{a}^{-1} \Gamma_{a} \sigma_{a}=B$, where $B$ is the group of integer translations. As a transformation on $H$ it is determined up to composition with a translation from the right. For a cusp $a$ and a multiplier system $\nu$ one defines $\chi_{a}$ by

$$
\nu\left(\sigma_{a}\left(\begin{array}{ll}
1 & 1 \\
0 & 1
\end{array}\right) \sigma_{a}^{-1}\right)=e\left(-\chi_{a}\right), \quad 0 \leq \chi_{a}<1 .
$$

The cusp $a$ is said to be singular for the multiplier system $\nu$ if $\chi_{a}=0$. 
It is known that if $f$ is a Maass form of weight $1 / 2$ for $\Gamma_{0}(4 N)$, then at every cusp $a$ of $\Gamma_{0}(4 N)$ it has a Fourier development of the form

$\left.f\right|_{\sigma_{a}}(z)=c_{f, a}(y)+\sum_{\substack{n=-\infty \\ n-\chi_{a} \neq 0}}^{\infty} b_{f, a}(n) W_{\frac{1}{4} \operatorname{sgn}\left(n-\chi_{a}\right), i t}\left(4 \pi\left|n-\chi_{a}\right| y\right) e\left(\left(n-\chi_{a}\right) x\right)$,

where $W$ is the Whittaker function, $1 / 4+t^{2}=-\lambda$, and $c_{f, a}(y)=0$ if $\chi_{a} \neq 0$, while for $\chi_{a}=0$ it is a linear combination of $y^{1 / 2+i t}$ and $y^{1 / 2-i t}$. The numbers $b_{f, a}(n)$ are called the Fourier coefficients of $f$ at the cusp $a$.

A.2. The operator $L$ and its action on Fourier coefficients. Let (up to Section A.4) $N$ be an odd positive integer. The three relevant cusps of $\Gamma_{0}(4 N)$ are $\infty,(N+1) /(4 N)$ and $(3 N+1) /(12 N)$. Now $\Gamma_{\infty}$ is obviously the group generated by $\left(\begin{array}{ll}1 & 1 \\ 0 & 1\end{array}\right)$, and it is not hard to check that

$$
\begin{aligned}
\gamma_{(N+1) /(4 N)} & =\left(\begin{array}{cc}
1-N(N+1) & (N+1)^{2} / 4 \\
-4 N^{2} & 1+N(N+1)
\end{array}\right), \\
\gamma_{(3 N+1) /(12 N)} & =\left(\begin{array}{cc}
1-3 N(3 N+1) & (3 N+1)^{2} / 4 \\
-36 N^{2} & 1+3 N(3 N+1)
\end{array}\right)
\end{aligned}
$$

are generators of $\Gamma_{(N+1) /(4 N)}$ and $\Gamma_{(3 N+1) /(12 N)}$, respectively.

For scaling matrices we can choose

$$
\begin{gathered}
\sigma_{\infty}=\left(\begin{array}{ll}
1 & 1 \\
0 & 1
\end{array}\right), \quad \sigma_{(N+1) /(4 N)}=\left(\begin{array}{cc}
(1+N) / 2 & 1 / 2 \\
2 N & 2
\end{array}\right), \\
\sigma_{(3 N+1) /(12 N)}=\left(\begin{array}{cc}
(1+3 N) / 2 & 1 / 2 \\
6 N & 2
\end{array}\right) .
\end{gathered}
$$

In our case

$$
\begin{gathered}
\nu\left(\gamma_{(N+1) /(4 N)}\right)=\bar{\varepsilon}_{1+N(N+1)}\left(\frac{-4 N^{2}}{1+N(N+1)}\right), \\
\nu\left(\gamma_{(3 N+1) /(12 N)}\right)=\bar{\varepsilon}_{1+3 N(3 N+1)}\left(\frac{-36 N^{2}}{1+3 N(3 N+1)}\right) .
\end{gathered}
$$

This shows that using the notations

$$
\begin{aligned}
& q= \begin{cases}(N+1) /(4 N) & \text { if } N \equiv 1(\bmod 4), \\
(3 N+1) /(12 N) & \text { if } N \equiv 3(\bmod 4),\end{cases} \\
& s= \begin{cases}(3 N+1) /(12 N) & \text { if } N \equiv 1(\bmod 4), \\
(N+1) /(4 N) & \text { if } N \equiv 3(\bmod 4),\end{cases}
\end{aligned}
$$

we have $\chi_{s}=0, \chi_{q}=3 / 4$ (so $s$ is a singular cusp of $\Gamma_{0}(4 N)$ for $\nu$, and $q$ is nonsingular). 
If $f$ is a function on $H$ with $f \mid \gamma=\nu(\gamma) f$ for every $\gamma \in \Gamma_{0}(4 N)$, let

$$
\begin{aligned}
L f & =\frac{1}{2(1+i)} \sum_{w(\bmod 4)}\left(f \mid\left(\begin{array}{cc}
1 & 1 / 4 \\
0 & 1
\end{array}\right)\right) \mid\left(\begin{array}{cc}
1 & 0 \\
4 N w & 1
\end{array}\right) \\
& =\frac{1}{2(1+i)} \sum_{w(\bmod 4)} f \mid\left(\begin{array}{cc}
1+N w & 1 / 4 \\
4 N w & 1
\end{array}\right) .
\end{aligned}
$$

It is easy to check that the definition is correct and the second equation holds.

Lemma A.2. Let $f$ be a function on $H$ with $f \mid \gamma=\nu(\gamma) f$ for every $\gamma \in \Gamma_{0}(4 N)$. Then the same is true for $L f$. The operator $L: V \rightarrow V$ is selfadjoint $\left(V=V_{1 / 2}(4 N)\right)$, commutes with $\Delta_{1 / 2}$ and with the Hecke operators $T_{p^{2}}$, and satisfies the quadratic equation $(L-1)(L+1 / 2)=0$. We have $V=$ $V^{+} \oplus V^{-}$(orthogonal decomposition), where $V^{+}=V_{1 / 2}^{+}(4 N)$ is the eigenspace of $L$ with eigenvalue 1 , and $V^{-}=V_{1 / 2}^{-}(4 N)$ is the eigenspace of $L$ with eigenvalue $-1 / 2$. For a Maass form $f \in V$, we have $f \in V^{+}$if and only if $b_{f, \infty}(n)=0$ for $n \equiv 2,3(\bmod 4)$.

Proof. In the holomorphic case these statements are proved in [Ko3], and the modifications needed are not hard.

We now describe the action of $L$ on Fourier coefficients.

Lemma A.3. Let $f$ be a Maass form of weight $1 / 2$. For $n \neq 0$ we have

$$
b_{L f, \infty}(n)= \begin{cases}\frac{1}{2} b_{f, \infty}(n)+\frac{1}{2(1+i)} b_{f, s}\left(\frac{n}{4}\right) & \text { if } n \equiv 0(\bmod 4), \\ \frac{1}{2} b_{f, \infty}(n)+\frac{1}{2(1+i)} b_{f, q}\left(\frac{n+3}{4}\right) & \text { if } n \equiv 1(\bmod 4), \\ -\frac{1}{2} b_{f, \infty}(n) & \text { if } n \equiv 2,3(\bmod 4) .\end{cases}
$$

Proof. For $w=2$ in (A.1) we have

$$
\left(\begin{array}{cc}
1+2 N & 1 / 4 \\
8 N & 1
\end{array}\right)=\left(\begin{array}{cc}
1+2 N & -(1+3 N) / 2 \\
8 N & 1-6 N
\end{array}\right)\left(\begin{array}{cc}
1 & 3 / 4 \\
0 & 1
\end{array}\right) .
$$

The first factor on the right-hand side is in $\Gamma_{0}(4 N), 1+2 N \equiv-1(\bmod 4)$, and $\left(\frac{8 N}{1+2 N}\right)=\left(\frac{-4}{1+2 N}\right)$, which is -1 . So the terms $w=0$ and $w=2$ in the expression (A.1) for $L f$ give

$$
\frac{1}{2(1+i)}\left(f\left|\left(\begin{array}{cc}
1 & 1 / 4 \\
0 & 1
\end{array}\right)+i f\right|\left(\begin{array}{cc}
1 & 3 / 4 \\
0 & 1
\end{array}\right)\right)
$$

while the terms $w=1$ and $w=3$ give

$$
\frac{1}{2(1+i)}\left(f\left|\sigma_{s}+f\right| \sigma_{q}\right) \mid\left(\begin{array}{cc}
2 & 0 \\
0 & 1 / 2
\end{array}\right) .
$$

From these last results we obtain the statement of the lemma. 
A.3. Kloosterman sums. We now determine (Lemma A.6 below) the relevant Kloosterman sums. In general, if $a_{1}, a_{2}$ are cusps of a group $\Gamma, \nu$ is a given multiplier system of weight $1 / 2$ on $\Gamma, m$ and $n$ are nonzero integers and $c>0$, then the definition of the Kloosterman sum is

$$
S_{a_{1}, a_{2}}^{\Gamma}(m, n ; c)=\sum_{\gamma=\left(\begin{array}{c}
a * \\
c d
\end{array}\right)} \bar{\nu}_{a_{1}, a_{2}}(\gamma) e\left(\frac{\left(m-\chi_{a_{1}}\right) a+\left(n-\chi_{a_{2}}\right) d}{c}\right) ;
$$

here the summation is over inequivalent elements $\gamma$ of $\sigma_{a_{1}}^{-1} \Gamma \sigma_{a_{2}}$ (in our case $\Gamma=\Gamma_{0}(4 N)$ ) with lower left entry $c$, where $\gamma_{1}$ and $\gamma_{2}$ are equivalent if $B \gamma_{1} B=B \gamma_{2} B$; and $\nu_{a_{1}, a_{2}}(\gamma)=\nu\left(\sigma_{a_{1}} \gamma \sigma_{a_{2}}^{-1}\right) w\left(\sigma_{a_{1}} \gamma \sigma_{a_{2}}^{-1}, \sigma_{a_{2}}\right) \bar{w}\left(\sigma_{a_{1}}, \gamma\right)$. We need $S_{\infty, \infty}^{\Gamma_{0}(4 N)}(m, n ; c), S_{\infty, s}^{\Gamma_{0}(4 N)}(m, n ; c)$ and $S_{\infty, q}^{\Gamma_{0}(4 N)}(m, n ; c)$.

If $a_{1}=\infty$, we can choose $\sigma_{a_{1}}=\left(\begin{array}{ll}1 & 0 \\ 0 & 1\end{array}\right)$, and then, since $c>0$, using Lemma A.1(i) we see that the $w$ functions are 1 provided the lower left entry of $\sigma_{a_{2}}$ is positive; but in our cases this condition holds, so $\nu_{a_{1}, a_{2}}(\gamma)=$ $\nu\left(\sigma_{a_{1}} \gamma \sigma_{a_{2}}^{-1}\right)$.

In preparation of Lemma A.6 we need two lemmas.

Lemma A.4. Suppose $M$ is an odd positive integer, $N \mid M$, and $a, b, c, d$ are arbitrary (not necessarily integer) numbers with $a d-b c=1$; furthermore

$$
\left(\begin{array}{ll}
a & b \\
c & d
\end{array}\right)=\left(\begin{array}{ll}
A & B \\
C & D
\end{array}\right)\left(\begin{array}{cc}
(1+M) / 2 & 1 / 2 \\
2 M & 2
\end{array}\right) .
$$

Then a necessary and sufficient condition for $\left(\begin{array}{ll}A & B \\ C & D\end{array}\right) \in \Gamma_{0}(4 N)$ is $\{2 \| c, N|c, 2| a, 2 \mid d, a d \equiv 1(\bmod (c / 2))\} \quad$ in the case $M \equiv 3(\bmod 4)$; $\{2 \| c, N \mid c, a \equiv 0(\bmod 1), d \equiv 0(\bmod 1), a(d-c / 2) \equiv 1(\bmod c)\}$ in the case $M \equiv 1(\bmod 4)$.

Proof. First we seek the condition for $\left(\begin{array}{ll}A & B \\ C & D\end{array}\right) \in \Gamma_{0}(4)$. Since $\left(\begin{array}{ll}1 & 0 \\ 4 & 1\end{array}\right) \in$ $\Gamma_{0}(4)$, and

$$
\left(\begin{array}{ll}
1 & 0 \\
4 & 1
\end{array}\right)=\left(\begin{array}{cc}
(1+M) / 2 & 1 / 2 \\
2 M & 2
\end{array}\right)\left(\begin{array}{cc}
0 & -1 / 2 \\
2 & (1+M) / 2
\end{array}\right)
$$

we have $\left(\begin{array}{ll}A & B \\ C & D\end{array}\right) \in \Gamma_{0}(4)$ if and only if $2 b$ is an integer, $2|d, 2| c$ and $2 \mid(1+M) b-a$. If these conditions are fulfilled, then $a$ is an integer (because $2 b \mid(1+M) b), a d-1=(2 b) c / 2$ is odd, and so $2 \| c$ and $2 b$ is odd. This implies that $a$ is odd for $M \equiv 1(\bmod 4)$, and even for $M \equiv 3(\bmod 4)$. It is easy to get as necessary and sufficient conditions for $\left(\begin{array}{ll}A & B \\ C & D\end{array}\right) \in \Gamma_{0}(4)$ the conditions stated in the lemma without $N \mid c$. But $C=2 c-2 M d$, and $N \mid M$, so we get the lemma. 
Lemma A.5. If $M$ is an odd positive integer, $\delta=\left(\begin{array}{ll}A & B \\ C & D\end{array}\right) \in \Gamma_{0}(4 N)$,

$$
\left(\begin{array}{ll}
a & b \\
c & d
\end{array}\right)=\left(\begin{array}{ll}
A & B \\
C & D
\end{array}\right)\left(\begin{array}{cc}
(1+M) / 2 & 1 / 2 \\
2 M & 2
\end{array}\right)
$$

and $c>0$, then

$$
\nu(\delta)= \begin{cases}\bar{\varepsilon}_{3 c / 2}\left(\frac{d / 2}{c / 2}\right) & \text { for } M \equiv 3(\bmod 4), \\ e\left(\frac{(c / 2)(d-c / 2-1)}{8}\right)\left(\frac{d}{c / 2}\right) & \text { for } M \equiv 1(\bmod 4) .\end{cases}
$$

Proof. We will repeatedly use the basic properties of the quadratic residue symbol (see Section A.1).

First observe that $\left(\frac{C}{D}\right)=\left(\frac{C+4 D}{-D}\right)$. Indeed, if $D<0$, we must have $C>0$ since $c>0$, and $\left(\frac{C+4 D}{-D}\right)=\left(\frac{C}{-D}\right)=\left(\frac{C}{D}\right)$. So we may assume $D>0$. Then $C+4 D>0$, as otherwise $2 c=(1+M)(C+4 D)-4 D \leq 0$, a contradiction. But then $\left(\frac{C+4 D}{-D}\right)=\left(\frac{C+4 D}{D}\right)=\left(\frac{C}{D}\right)$. We will use this observation twice. Observe also that $c \equiv 2(\bmod 4)$ and $d$ is an even integer.

Assume first that $M \equiv 3(\bmod 4)$. Then since $4 \mid 1+M$, we have

$$
\begin{aligned}
\left(\frac{d / 2}{c / 2}\right) & =\left(\frac{C / 4+D}{(1+M)(C / 4+D)-D}\right) \\
& =\left(\frac{C / 4+D}{-D}\right)=\left(\frac{C+4 D}{-D}\right)=\left(\frac{C}{D}\right) .
\end{aligned}
$$

On the other hand $\varepsilon_{D}=\varepsilon_{3 c / 2}$ since $4|C, 4| 1+M$, and this proves the lemma in this case.

For $M \equiv 1(\bmod 4)$ set $x=d-c / 2$. Then $x$ is an odd integer, and we will prove $\left(\frac{c}{x}\right)=\left(\frac{C}{D}\right)$. Indeed, $c>0$, so

$$
\left(\frac{c}{x}\right)=\left(\frac{c}{|x|}\right)=\left(\frac{2 d}{|x|}\right)=\left(\frac{d / 2}{|x|}\right) .
$$

We have $x=d-c / 2=(1-M) \frac{d}{2}+D$. If this is positive, then $d>0$ (as $c>0$ ), and then $D>0$ (since $1-M \leq 0$ ). In this case, continuing the computation, since $4 \mid 1-M$ and $d / 2=C / 4+D$, we have

$$
\left(\frac{d / 2}{x}\right)=\left(\frac{C / 4+D}{D}\right)=\left(\frac{C}{D}\right)
$$

If $x=d-c / 2<0$, then as above,

$$
\left(\frac{d / 2}{-x}\right)=\left(\frac{C / 4+D}{-D}\right)=\left(\frac{C}{D}\right)
$$

again (see the beginning of the proof). On the other hand $\varepsilon_{D}=\varepsilon_{d-c / 2}$ 
(again because $4 \mid 1-M)$, so by quadratic reciprocity $(c>0$ !)

$$
\nu(\delta)=\bar{\varepsilon}_{d-c / 2}\left(\frac{2}{d-c / 2}\right)(-1)^{(c / 2-1) / 2 \cdot(d-c / 2-1) / 2}\left(\frac{d-c / 2}{c / 2}\right) .
$$

It is easy to check that $\bar{\varepsilon}_{n}\left(\frac{2}{n}\right)=e\left(\frac{n-1}{8}\right)$ for every odd $n$, and this proves the lemma.

We can now turn to the main result of this section.

LEMMA A.6. We have

$$
\begin{gathered}
S_{\infty, \infty}^{\Gamma_{0}(4 N)}(m, n ; c)=0 \quad \text { if } c \text { is not divisible by } 4 N \\
S_{\infty, s}^{\Gamma_{0}(4 N)}(m, n ; c)=S_{\infty, q}^{\Gamma_{0}(4 N)}(m, n ; c)=0 \\
\text { if the condition }(2 \| c \text { and } N \mid c) \text { does not hold. }
\end{gathered}
$$

Moreover,

$$
S_{\infty, \infty}^{\Gamma_{0}(4 N)}(m, n ; c)=\sum_{\substack{x(\bmod c) \\(x, c)=1}} \varepsilon_{x}\left(\frac{c}{x}\right) e\left(\frac{m \bar{x}+n x}{c}\right) \quad \text { if } 4 N \mid c ;
$$

$S_{\infty, s}^{\Gamma_{0}(4 N)}(m, n ; c)=\varepsilon_{3 c / 2} \sum_{\substack{x(\bmod c / 2) \\(x, c / 2)=1}}\left(\frac{x}{c / 2}\right) e\left(\frac{(m \overline{4}) \bar{x}+n x}{c / 2}\right)$

(iii) $S_{\infty, q}^{\Gamma_{0}(4 N)}(m, n ; c)=\bar{\varepsilon}_{3 c / 2}(-1)^{n} \sum_{\substack{x \bmod c) \\(x, c)=1}}\left(\frac{x}{c / 2}\right) e\left(\frac{m \bar{x}+\left(n-V_{c}\right) x}{c}\right)$

$$
\text { if } 2 \| c \text { and } N \mid c \text {; }
$$

if $2 \| c$ and $N \mid c$,

where $V_{c}$ is the integer defined by

$$
V_{c}= \begin{cases}(6+c) / 8 & \text { for } c \equiv 2(\bmod 8), \\ (6+3 c) / 8 & \text { for } c \equiv 6(\bmod 8) .\end{cases}
$$

Proof. The statement for $S_{\infty, \infty}^{\Gamma_{0}(4 N)}(m, n ; c)$ is obvious, and the others are immediate consequences of the previous two lemmas, because $\sigma_{s}$ and $\sigma_{q}$

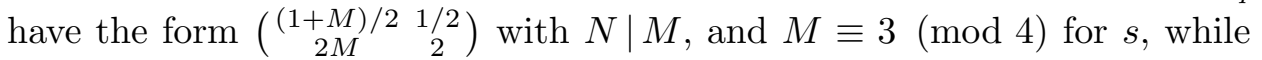
$M \equiv 1(\bmod 4)$ for $q$. In the case of $S_{\infty, s}^{\Gamma_{0}(4 N)}(m, n ; c)$ we write $x=d / 2$. In the case of $S_{\infty, q}^{\Gamma_{0}(4 N)}(m, n ; c)$ we write $x=d-c / 2$, use the fact that $x-1$ is even,

$$
e\left(\frac{(c / 2)(1-x)}{8}\right)=\bar{\varepsilon}_{3 c / 2} e\left(\frac{\left(3 / 4-V_{c}\right) x}{c}\right) e\left(\frac{3}{8}\right)
$$


for $2 \| c$, and

$$
e\left(\frac{(n-3 / 4) d}{c}\right)=e\left(\frac{(n-3 / 4) x}{c}\right)(-1)^{n} e\left(-\frac{3}{8}\right) .
$$

The following lemma gives another expression for the sums $S_{K}(m, n ; c)$ introduced in Section 3.

Lemma A.7. Let $4 \| c, m \equiv 0,1(\bmod 4), n \equiv 0,1(\bmod 4)$, and let

$$
\begin{gathered}
S_{\infty, \infty}(m, n ; c)=S_{\infty, \infty}^{\Gamma_{0}(4)}(m, n ; c), \\
S_{\infty, s}(m, n ; c)=S_{\infty, s}^{\Gamma_{0}(4)}(m, n ; c), \quad S_{\infty, q}(m, n ; c)=S_{\infty, q}^{\Gamma_{0}(4)}(m, n ; c),
\end{gathered}
$$

so their explicit definition is given in Lemma A.6 with $N=1$. Then

$S_{K}(m, n ; c)$

$$
= \begin{cases}S_{\infty, \infty}(m, n ; c)+\frac{2}{1+i} S_{\infty, s}\left(m, \frac{n}{4} ; \frac{c}{2}\right) & \text { if } n \equiv 0(\bmod 4), \\ S_{\infty, \infty}(m, n ; c)+\frac{2}{1+i} S_{\infty, q}\left(m, \frac{n+3}{4} ; \frac{c}{2}\right) & \text { if } n \equiv 1(\bmod 4) .\end{cases}
$$

In other words, we have

$$
S_{\infty, \infty}(m, n ; c)= \begin{cases}(1-i) S_{\infty, s}\left(m, \frac{n}{4} ; \frac{c}{2}\right) & \text { for } n \equiv 0(\bmod 4), \\ (1-i) S_{\infty, q}\left(m, \frac{n+3}{4} ; \frac{c}{2}\right) & \text { for } n \equiv 1(\bmod 4) .\end{cases}
$$

Pr o of. By quadratic reciprocity, using the pairing $x \leftrightarrow x+c / 2$, the fact that if $x \equiv 1(\bmod 4)$, then $x+c / 2 \equiv 3(\bmod 4)$, and that $\overline{x+c / 2} \equiv \bar{x}+c / 2$ $(\bmod c)$, we have

$$
\begin{aligned}
& S_{\infty, \infty}(m, n ; c) \\
& \quad=\left(1+i(-1)^{(c / 4-1) / 2+m+n}\right) \sum_{\substack{y \equiv 1(\bmod 4), y(\bmod c / 4) \\
(y, c / 4)=1}}\left(\frac{y}{c / 4}\right) e\left(\frac{m \bar{y}+n y}{c}\right),
\end{aligned}
$$

where $y \bar{y} \equiv 1(\bmod c)$. Since

$$
e\left(\frac{m \bar{y}}{c}\right)=e\left(\frac{m \overline{4} \bar{y}}{c / 4}\right) e\left(\frac{m c / 4}{4}\right) \quad \text { for } y \equiv 1(\bmod 4),
$$

and for $m \equiv 0,1(\bmod 4)$

$$
\left(1+i(-1)^{(c / 4-1) / 2+m}\right) e\left(\frac{m c / 4}{4}\right)=(1-i) \varepsilon_{3 c / 4},
$$

in the case $n \equiv 0(\bmod 4)$ we have

$$
S_{\infty, \infty}(m, n ; c)=(1-i) S_{\infty, s}(m, n / 4 ; c / 2) .
$$


If $n \equiv 1(\bmod 4)$ take an integer $r$ with $r \equiv 2(\bmod c / 4), r \equiv 1$ $(\bmod 4)$, and let $r \bar{r} \equiv 1(\bmod c)$. Then for $y \equiv 1(\bmod 4)$ we have

$$
e\left(\frac{n y}{c}\right)=e\left(\frac{\left((n+3) / 4-V_{c / 2}\right) r y}{c / 2}\right)(-1)^{(n+3) / 4-V_{c / 2}} e\left(\frac{4 V_{c / 2}-3}{c} y\right),
$$

and

$$
e\left(\frac{m \bar{y}}{c}\right)=e\left(\frac{m \overline{r y}}{c / 2}\right) e\left(-\frac{m c / 4}{4}\right)
$$

because after writing every term in the form $e(l / c)$ with some integer $l$ we have to prove a congruence $\bmod c$, which is easily proved $\bmod 4$ and $\bmod c / 4$ (and $(4, c / 4)=1)$ by our conditions.

Since

$$
e\left(\frac{4 V_{c / 2}-3}{c} y\right)=i(-1)^{(c / 4-1) / 2}
$$

for $y \equiv 1(\bmod 4)$, so by the substitution $r y \mapsto y$, using

$$
\left(\frac{2}{c / 4}\right)(-1)^{V_{c / 2}}(-1)^{(c / 4-1) / 2}=-1,
$$

and the conjugate of (A.2), we get

$$
S_{\infty, \infty}(m, n ; c)=(1-i) S_{\infty, q}(m,(n+3) / 4 ; c / 2) \quad \text { for } n \equiv 1(\bmod 4) .
$$

A.4. Kuznetsov's formula. First we state Kuznetsov's sum formula for the weight $k=1 / 2$ and for the group $\Gamma_{0}(4 N)$ ( $N$ may be even here) as given in $[\mathrm{P}]$ (see also $[\mathrm{Du}]$ ). Proskurin actually derived the formula for more general groups and weights, but considered only the case when the two cusps are equal. The modifications for mixed cusps are straightforward. We will need the case of mixed cusps, so state the formula in such a form.

Let $N$ be a (not necessarily odd) positive integer. In addition to the notations introduced in Section 3, let $a_{1}, a_{2}$ be two cusps of $\Gamma_{0}(4 N)$, and let $\left\{f_{i j}\right\}_{i=1}^{d_{j}}$ be an orthonormal basis for the space of holomorphic cusp forms for $\Gamma_{0}(4 N)$ of weight $1 / 2+2 j(j=1,2, \ldots)$, with multiplier system $\nu$, and with Fourier coefficients $b_{i j, a}(n)$ at a cusp $a$.

For $n, m \geq 1$ set

$$
\begin{aligned}
V_{1}(m, n)= & 4 \sqrt{\left(m-\chi_{a_{1}}\right)\left(n-\chi_{a_{2}}\right)} \sum_{j} \frac{\bar{b}_{j, a_{1}}(m) b_{j, a_{2}}(n)}{\operatorname{ch}\left(\pi t_{j}\right)} \widehat{\varphi}\left(t_{j}\right), \\
V_{2}(m, n)= & \sum_{j=1}^{h} \int_{-\infty}^{\infty}\left(\frac{n-\chi_{a_{2}}}{m-\chi_{a_{1}}}\right)^{i t} \\
& \times \bar{\varphi}_{j, m, a_{1}}(1 / 2+i t) \varphi_{j, n, a_{2}}(1 / 2+i t) \frac{\widehat{\varphi}(t)}{\operatorname{ch}(\pi t)|\Gamma(3 / 4+i t)|^{2}} d t
\end{aligned}
$$




$$
\begin{aligned}
V_{3}(m, n)= & 4 \sum_{j \geq 1} \frac{\Gamma(1 / 2+2 j) e(1 / 8+j / 2)}{(4 \pi)^{1 / 2+2 j}\left(m-\chi_{a_{1}}\right)^{j-1 / 4}\left(n-\chi_{a_{2}}\right)^{j-1 / 4}} \\
& \times \widetilde{\varphi}(1 / 2+2 j) \sum_{i=1}^{d_{j}} \bar{b}_{i j, a_{1}}(m) b_{i j, a_{2}}(n) .
\end{aligned}
$$

Lemma A.8 (Proskurin). With the above notations, for $n, m \geq 1$ we have

$$
\sum_{c>0} \frac{S_{a_{1}, a_{2}}^{\Gamma_{0}(4 N)}(m, n ; c)}{c} \varphi\left(4 \pi \frac{\sqrt{\left(m-\chi_{a_{1}}\right)\left(n-\chi_{a_{2}}\right)}}{c}\right)=\sum_{l=1}^{3} V_{l}(m, n) .
$$

Proof of Theorem B. The case $2 \mid N$ is a straightforward application of Kuznetsov's formula (Lemma A.8), so we assume that $N$ is odd. By Lemma A.7 we have

$$
\begin{aligned}
\sum= & \sum_{\substack{c>0 \\
N \mid c}} \frac{S_{\infty, \infty}(m, n ; c)}{c} \varphi\left(4 \pi \frac{\sqrt{m n}}{c}\right) \\
& +\frac{1}{1+i} \sum_{\substack{c>0 \\
N \mid c}} \frac{S_{\infty, s}(m, n / 4 ; c)}{c} \varphi\left(4 \pi \frac{\sqrt{m(n / 4)}}{c}\right),
\end{aligned}
$$

if $n \equiv 0(\bmod 4)$, and

$$
\begin{aligned}
\sum= & \sum_{\substack{c>0 \\
N \mid c}} \frac{S_{\infty, \infty}(m, n ; c)}{c} \varphi\left(4 \pi \frac{\sqrt{m n}}{c}\right) \\
& +\frac{1}{1+i} \sum_{\substack{c>0 \\
N \mid c}} \frac{S_{\infty, q}(m,(n+3) / 4 ; c)}{c} \varphi\left(4 \pi \frac{\sqrt{m\left((n+3) / 4-\chi_{q}\right)}}{c}\right),
\end{aligned}
$$

if $n \equiv 1(\bmod 4)$.

Now we apply Kuznetsov's formula three times, and use Lemma A.3, to get

$$
\sum=4 \sqrt{m n} \sum_{j} \frac{\bar{b}_{j, \infty}(m)\left\{\frac{1}{2} b_{j, \infty}(n)+b_{L f_{j}, \infty}(n)\right\}}{\operatorname{ch}\left(\pi t_{j}\right)} \widehat{\varphi}\left(t_{j}\right)+E .
$$

This proves the theorem, because $\frac{1}{2} b_{j, \infty}(n)+b_{L f_{j}, \infty}(n)$ is 0 for $f_{j} \in V^{-}$, and $\frac{3}{2} b_{j, \infty}(n)$ for $f_{j} \in V^{+}$.

A.5. Inverting the formula. The following lemma is important in inverting the Kuznetsov transform $\varphi \mapsto \widehat{\varphi}$. This lemma is in a sense the converse of the theorem in the Appendix of $[\mathrm{K}]$. We need only the case $\alpha=-1 / 2$ here. 
Lemma A.9. Let $h$ be an even smooth function on $(-\infty, \infty)$, and assume that for some $\varepsilon>0$ we have $h^{(l)}(t)=O_{l}\left(e^{\pi t} t^{-(2+\varepsilon)}\right)$ as $t \rightarrow \infty$ for $l=$ $0,1,2, \ldots$ Define

$$
F(x, t, \alpha)=J_{i t}(x) \cos \frac{\pi}{2}(\alpha-i t)-J_{-i t}(x) \cos \frac{\pi}{2}(\alpha+i t) .
$$

Then for any $s>0$ we have

$$
h(s)=-\int_{0}^{\infty} F(x, s,-1 / 2)\left(\int_{0}^{\infty} \frac{F(x, t,-1 / 2)}{\operatorname{ch} \pi t} h(t) \frac{t d t}{\operatorname{sh} \pi t}\right) \frac{d x}{x} .
$$

Proof. First we assume that $h(t)=0$ for $|t| \geq T$ with some positive $T$. The lemma will follow from this by approximating a general $h(t)$ by $h(t) \omega_{0}(t / T)$ with $T \rightarrow \infty$, where $\omega_{0}$ is a fixed smooth function on $(-\infty, \infty)$ with $0 \leq \omega_{0} \leq 1, \omega_{0}(t)=1$ for $|t| \leq 1 / 2$, and $\omega_{0}(t)=0$ for $|t| \geq 1$. Using the formula

$$
J_{i t}(x)=\frac{(x / 2)^{i t}}{\Gamma(1 / 2+i t) \Gamma(1 / 2)} \int_{-1}^{1}\left(1-s^{2}\right)^{i t-1 / 2} \cos s x d s
$$

we see that if we know the theorem for functions with compact support, this approximation gives the theorem for any $h$ satisfying the conditions of the lemma (for $x$ small we replace $\cos s x$ by 1 in (A.3), and then we apply partial integration two times in $t$ ).

So let $h(t)=0$ for $|t| \geq T$. It is easy to see by (A.3) that the integral in $x$ is absolutely convergent. Let $g_{n}(y)$ be the function on $(-\infty, \infty)$ defined by $g_{n}(y)=0$ for $y \leq a_{n}, g_{n}(y)=1$ for $y \geq b_{n}, g_{n}(y)=\left(y-a_{n}\right) /\left(b_{n}-a_{n}\right)$ for $a_{n} \leq y \leq b_{n}$, where $\left\{a_{n}\right\}$ and $\left\{b_{n}\right\}$ are two sequences with $\lim _{n \rightarrow \infty} b_{n}=-\infty$ and $\lim _{n \rightarrow \infty}\left(b_{n}-a_{n}\right)=\infty$. Then it is enough to prove that

$$
h(s)=-\lim _{n \rightarrow \infty} \int_{0}^{\infty} g_{n}(\log x) F(x, s,-1 / 2)\left(\int_{0}^{\infty} \frac{F(x, t,-1 / 2)}{\operatorname{ch} \pi t} h(t) \frac{t d t}{\operatorname{sh} \pi t}\right) \frac{d x}{x},
$$

so that

$$
h(s)=-\lim _{n \rightarrow \infty} \int_{0}^{\infty}\left(\int_{0}^{\infty} g_{n}(\log x) F(x, s,-1 / 2) F(x, t,-1 / 2) \frac{d x}{x}\right) \frac{h(t)}{\operatorname{ch} \pi t} \frac{t d t}{\operatorname{sh} \pi t} .
$$

We now prove that for $\operatorname{Re} \nu \geq 0, \nu \neq-i s$ we have

$$
\lim _{n \rightarrow \infty} \int_{0}^{\infty} g_{n}(\log x) J_{\nu}(x) J_{i s}(x) \frac{d x}{x}=\frac{2 \sin \pi(\nu-i s) / 2}{\pi(\nu-i s)(\nu+i s)} .
$$

Indeed, for $\operatorname{Re} \nu>0$ this follows from (B.37) of [I1], so it is enough to prove that the limit also exists for $\operatorname{Re} \nu=0, \nu \neq-i s$, and gives a continuous function of $\nu$ for $\operatorname{Re} \nu \geq 0, \nu \neq-i s$. To prove this we can integrate from 0 to 1 , and here we can replace the $J$-functions with the first term of the power 
series expansion, obtaining $\int_{0}^{1} g_{n}(\log x) x^{\nu+i s} d x / x$. Applying the substitution $e^{y}=x$ and then integrating by parts we get

$$
\int_{0}^{1} g_{n}(\log x) x^{\nu+i s} \frac{d x}{x}=\frac{1}{\nu+i s}-\frac{1}{b_{n}-a_{n}} \int_{a_{n}}^{b_{n}} \frac{e^{y(\nu+i s)}}{\nu+i s} d y .
$$

The limit of this as $n \rightarrow \infty$ is $1 /(\nu+i s)$ (see the conditions on $a_{n}$ and $b_{n}$ ), which proves the present assertion.

Then an easy calculation shows that for $t, s>0, t \neq s$, we have

$$
\lim _{n \rightarrow \infty} \int_{0}^{\infty} g_{n}(\log x) F(x, s,-1 / 2) F(x, t,-1 / 2) \frac{d x}{x}=0 .
$$

Let $s$ and $t$ be bounded, $t>0, s>0, t \neq s$. Then

$$
\begin{aligned}
\int_{0}^{\infty} g_{n}(\log x) F & (x, s,-1 / 2) F(x, t,-1 / 2) \frac{d x}{x} \\
= & \lim _{N \rightarrow \infty} \int_{0}^{\infty}\left(g_{n}-g_{N}\right)(\log x) F(x, s,-1 / 2) F(x, t,-1 / 2) \frac{d x}{x}
\end{aligned}
$$

showing by (A.4) that

$$
\int_{0}^{\infty} g_{n}(\log x) F(x, s,-1 / 2) F(x, t,-1 / 2) \frac{d x}{x}=\sum R_{n}( \pm t, \pm s)+o_{n}(1),
$$

where

$$
\begin{aligned}
R_{n}(T, S)= & \frac{2^{-i(T+S)} \cos \frac{\pi}{2}(-1 / 2-i T) \cos \frac{\pi}{2}(-1 / 2-i S)}{\Gamma(1+i T) \Gamma(1+i S)} \\
& \times(-1)^{1+T /|T|+S /|S|} \frac{1}{b_{n}-a_{n}} \int_{a_{n}}^{b_{n}} \frac{e^{i y(T+S)}}{i(T+S)} d y
\end{aligned}
$$

and since

$$
\lim _{y \rightarrow-\infty} \int_{0}^{\infty} H(t) \frac{\sin y(t-s)}{t-s} d t=-H(s) \int_{-\infty}^{\infty} \frac{\sin x}{x} d x=-\pi H(s)
$$

for any smooth, compactly supported function $H$ on $[0, \infty)$ and for any $s>0$, and

$\frac{\pi s}{\Gamma(1+i s) \Gamma(1-i s)}=\operatorname{sh} \pi s, \quad 2 \cos \frac{\pi}{2}\left(-\frac{1}{2}-i s\right) \cos \frac{\pi}{2}\left(-\frac{1}{2}+i s\right)=\operatorname{ch} \pi s$,

this proves the lemma.

Corollary. Let $f$ be an even smooth function on $(-\infty, \infty)$, and assume that $f^{(l)}(t)=O_{l}\left(t^{-(3 / 2+\varepsilon)}\right)$ for some $\varepsilon>0$ as $t \rightarrow \infty$ for $l=0,1,2, \ldots$ 
Define

$\varphi(x)=-\frac{2}{\pi^{2} e(3 / 8)} \int_{-\infty}^{\infty} \frac{J_{2 i t}(x) \cos (\pi i t+\pi / 4) f(t) \Gamma(1 / 4+i t) \Gamma(1 / 4-i t)}{\operatorname{ch} \pi t} t d t$,

and assume that $\varphi(0)=\varphi^{(1)}(0)=0$, and $\varphi(x)=O\left(x^{-1-\varepsilon}\right)$ and $\varphi^{(l)}(x)=$ $O\left(x^{-2-\varepsilon}\right)$ for $l=1,2,3$ as $x \rightarrow \infty$. Then Theorem $B$ is applicable to this $\varphi$, i.e. $\widetilde{\varphi}(1 / 2+2 j)=0$ for $j=1,2, \ldots$, and $f=\widehat{\varphi}$.

Proof. The condition $\widetilde{\varphi}(1 / 2+2 j)=0$ for $j=1,2, \ldots$ follows from (B.37) of [I1], and $f=\widehat{\varphi}$ by Lemma A.9 with

$$
h(t)=\frac{(\operatorname{sh} \pi t)(\operatorname{ch} \pi t)}{\operatorname{ch}(\pi t / 2)} \Gamma\left(\frac{1}{4}+i \frac{t}{2}\right) \Gamma\left(\frac{1}{4}-i \frac{t}{2}\right) f\left(\frac{t}{2}\right)
$$

and the definition of the Kuznetsov transform.

\section{References}

[A-L] A. O. L. Atkin and J. Lehner, Hecke operators on $\Gamma_{0}(m)$, Math. Ann. 185 (1970), 134-160.

[B] A. Biró, On a generalization of the Selberg trace formula, Acta Arith. 87 (1999), 319-338.

[Da] H. Davenport, Multiplicative Number Theory, 2nd ed., Springer, Berlin, 1980.

[Du] W. Duke, Hyperbolic distribution problems and half-integral weight Maass forms, Invent. Math. 92 (1988), 73-90.

[G] J. Guo, On the positivity of the central critical values of automorphic L-functions for GL(2), Duke Math. J. 83 (1996), 157-190.

[I1] H. Iwaniec, Introduction to the Spectral Theory of Automorphic Forms, Rev. Mat. Iberoamericana, 1995.

[I2] - Topics in Classical Automorphic Forms, Lecture Notes, Rutgers University, 1995.

[K-S] S. Katok and P. Sarnak, Heegner points, cycles and Maass forms, Israel J. Math. 84 (1993), 193-227.

[Ko1] W. Kohnen, Modular forms of half-integral weight on $\Gamma_{0}(4)$, Math. Ann. 248 (1985), 249-266.

[Ko2] - Fourier coefficients of modular forms of half-integral weight, ibid. 271 (1985), 237-268.

[Ko3] —, Newforms of half-integral weight, J. Reine Angew. Math. 333 (1982), 32-72.

[Ko-Z] W. Kohnen and D. Zagier, Values of L-series of modular forms at the center of the critical strip, Invent. Math. 64 (1981), 175-198.

[K] N. V. Kuznetsov, Petersson's conjecture for cusp forms of weight 0 and Linnik's conjecture. Sums of Kloosterman sums, Mat. Sb. 111 (1980), 334-383 (in Russian).

[La] E. Landau, Vorlesungen über Zahlentheorie, 3 vol., Hirzel, Leipzig, 1927.

[Le] N. N. Lebedev, Special Functions and their Applications, 2nd ed., Moscow, 1963.

[M] H. Maass, Über die räumliche Verteilung der Punkte in Gittern mit indefiniter Metrik, Math. Ann. 138 (1959), 287-315. 
[M-O] O. Magnus and F. Oberbettinger, Formulas and Theorems for the Functions of Mathematical Physics, Springer, Berlin, 1966.

[N] S. Niwa, Modular forms of half-integral weight and the integral of certain theta functions, Nagoya Math. J. 56 (1974), 147-161.

[P] N. V. Proskurin, General Kloosterman sums, preprint LOMI R-3-80, Akad. Nauk SSSR Mat. Inst. Leningr. Otd. (1980) (in Russian), MR 82E 10040.

[P-Sz] G. Pólya and G. Szegö, Problems and Theorems in Analysis, Vol. 1, Springer, Berlin, 1972.

[Shim] G. Shimura, On modular forms of half-integral weight, Ann. of Math. (2) 97 (1973), 440-481.

[Shin] T. Shintani, On construction of holomorphic cusp forms of half-integral weight, Nagoya Math. J. 58 (1975), 83-126.

[Wal] J. L. Waldspurger, Sur les coefficients de Fourier des formes modulaires de poids demi-entier, J. Math. Pures Appl. 60 (1981), 375-484.

[W] G. N. Watson, A Treatise on the Theory of Bessel Functions, 2nd ed., Cambridge Univ. Press, 1952.

Mathematical Institute

of the Hungarian Academy of Sciences

Realtanoda u. 13-15

H-1053 Budapest, Hungary

E-mail: biroand@math-inst.hu

Received on 15.9.1997

and in revised form on 4.6.1999 